\title{
FOLLOWING BLACK HOLE SCALING RELATIONS THROUGH GAS-RICH MERGERS
}

\author{
Anne M. Medling ${ }^{1}$, Vivian U ${ }^{2}$, Claire E. Max ${ }^{3}$, David B. Sanders ${ }^{4}$, Lee Armus ${ }^{5}$, Bradford Holden ${ }^{3}$, Etsuko Mieda ${ }^{6}$, \\ SHElley A. WRight ${ }^{6,7}$, AND JAMES E. LARKIN ${ }^{8}$ \\ ${ }^{1}$ Research School of Astronomy \& Astrophysics, Mount Stromlo Observatory, Australia National University, \\ Cotter Road, Weston, ACT 2611, Australia; anne.medling@anu.edu.au \\ ${ }^{2}$ Department of Physics and Astronomy, University of California, Riverside, 900 University Avenue, Riverside, CA 92521, USA \\ ${ }^{3}$ Department of Astronomy \& Astrophysics, University of California, Santa Cruz, 1156 High Street, Santa Cruz, CA 95064, USA \\ ${ }^{4}$ Institute for Astronomy, University of Hawaii, 2680 Woodlawn Drive, Honolulu, HI 96822, USA \\ ${ }^{5}$ Spitzer Science Center, California Institute of Technology, 1200 E. California Blvd., Pasadena, CA 91125, USA \\ ${ }^{6}$ Department of Astronomy \& Astrophysics, University of Toronto, 50 St. George Street. Toronto, ON M5S 3H4, Canada \\ ${ }^{7}$ Dunlap Institute for Astronomy \& Astrophysics, 50 St. George Street. Toronto, ON M5S 3H4, Canada \\ ${ }^{8}$ Department of Physics and Astronomy, University of California, Los Angeles, CA 90095-1547, USA \\ Received 2014 September 22; accepted 2015 February 3; published 2015 April 16
}

\begin{abstract}
We present black hole mass measurements from kinematic modeling of high-spatial resolution integral field spectroscopy of the inner regions of nine nearby (ultra-)luminous infrared galaxies in a variety of merger stages. These observations were taken with OSIRIS and laser guide star adaptive optics on the Keck I and Keck II telescopes, and reveal gas and stellar kinematics inside the spheres of influence of these supermassive black holes. We find that this sample of black holes are overmassive $\left(\sim 10^{7-9} M_{\odot}\right)$ compared to the expected values based on black hole scaling relations, and suggest that the major epoch of black hole growth occurs in early stages of a merger, as opposed to during a final episode of quasar-mode feedback. The black hole masses presented are the dynamical masses enclosed in $\sim 25 \mathrm{pc}$, and could include gas which is gravitationally bound to the black hole but has not yet lost sufficient angular momentum to be accreted. If present, this gas could in principle eventually fuel active galactic nucleus feedback or be itself blown out from the system.
\end{abstract}

Key words: galaxies: evolution - galaxies: interactions - galaxies: kinematics and dynamics - galaxies: nuclei

\section{INTRODUCTION}

Virtually every massive galaxy hosts a supermassive black hole in its core. These central black holes exhibit tight correlations with properties of their host galaxies' bulges (Kormendy et al. 2001): bulge mass (Kormendy \& Richstone 1995; Magorrian et al. 1998), bulge luminosity (Marconi \& Hunt 2003), and bulge stellar velocity dispersion (Ferrarese $\&$ Merritt 2000; Gebhardt et al. 2000; Tremaine et al. 2002). Recently these correlations were re-explored using a wealth of new black hole mass measurements $\left(10^{6}-10^{10} M_{\odot}\right)$ and galaxy parameters by McConnell \& Ma (2013) and Graham \& Scott (2013). However, the discovery that the $M_{\mathrm{BH}}-\sigma_{*}$ relation may evolve with redshift (e.g., Zhang et al. 2012) has led to the suggestion that the most basic scaling relation may instead be with total stellar mass (Jahnke et al. 2009; Cisternas et al. 2011a); in this case, the evolution of the $M_{\mathrm{BH}}-\sigma_{*}$ relation with redshift indicates the changing fractions of mass in galaxy bulges (which contribute to $\sigma_{*}$ ) versus disks (which do not). See Kormendy \& Ho (2013) for a detailed review of our current understanding of black hole scaling relations.

The mechanism through which black hole masses correlate with galaxy properties has been canonically associated with gas-rich galaxy mergers (e.g., Hopkins et al. 2006). Gravitational torques funnel the gas into their centers, triggering two phenomena: an intense burst of star formation to feed the bulge, and accretion of gas onto the black holes in the centers of each galaxy. It has been postulated that black hole growth can regulate this process through active galactic nucleus (AGN) feedback (Springel et al. 2005a; Hopkins \& Elvis 2010) via massive winds that evacuate the gas from the galaxy on short timescales, cutting off star formation and future black hole growth. This sense of self-regulation has been confirmed observationally by Kauffmann \& Heckman (2009), who find that the Eddington ratio of a sample of AGNs depends on the supply of cold gas in the galaxy. If there is plenty of cold gas, the accretion rate does not depend on the quantity of gas available; if the supply of cold gas is limited, the accretion rate depends on the rate at which stellar winds provide fuel for the AGN. Though the detailed mechanisms causing these correlations are still unconfirmed, star formation and black hole growth are fed by the same reservoir of inflowing gas; their growth histories are intertwined. It is likely that these two processes compete for fuel in a predictable fashion.

To understand this interplay, it is critical to look at systems in the midst of this increased fueling. One set of such galaxies are gas-rich mergers, which tend to have extreme bursts of star formation and a higher incidence of AGN activity (e.g., Sanders et al. 1988; Sanders \& Mirabel 1996; Veilleux et al. 2002; Ishida 2004; Ellison et al. 2013; Koss et al. 2013). During such a merger, does the black hole grow first, leaving the stars to slowly consume the remaining gas? Or is star formation quenched once the black hole reaches a bright quasar phase of extreme growth? The position of a merger on black hole scaling relations would indicate the relative growth timescales, and confirm whether the putative quasar-mode feedback occurs at the end of a merger (see Figure 1 for the schematic example for the $M_{\mathrm{BH}}-\sigma_{*}$ relation). As star formation happens on few-kiloparsec spatial scales, accreting gas must lose less angular momentum to fuel a starburst than to feed a black hole. Subsequent models therefore suggest that a black hole would grow substantially only after star formation has quenched itself and the galaxy bulge is in place (Cen 2012), or at least that the peak black hole accretion time 


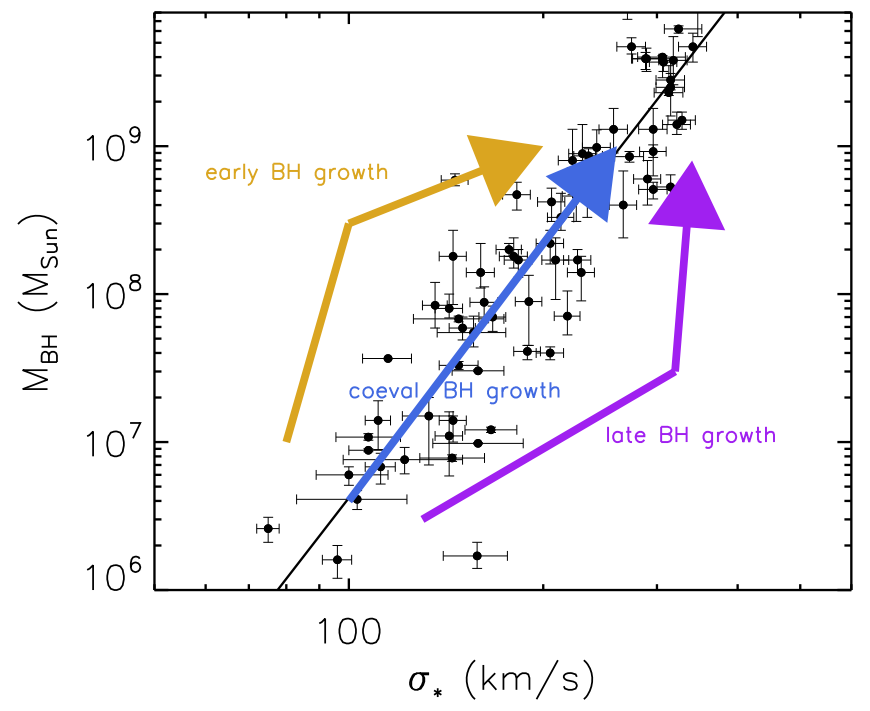

Figure 1. $M_{\mathrm{BH}}-\sigma_{*}$ relation for isolated galaxies from McConnell \& $\mathrm{Ma}$ (2013) (black) with three possible evolutionary tracks for merging galaxies overlaid. If the black hole grows first or more quickly than the galaxy bulge, mergers would lie above the relation (as shown by the gold arrows). If the black hole growth lags the bulge growth and is responsible for curtailing evolution (e.g., quenching through AGN feedback), mergers would lie below the relation (as shown by the purple arrows). If instead the black hole and the bulge grow in lockstep, the mergers would remain on the relation (as shown by the blue arrow).

occurs later in a merger relative to peak star formation (Hopkins 2012). This scenario would predict that gas-rich mergers would fall below black hole scaling relations.

Merger-driven galaxy evolution is not a complete explanation for all black hole growth, however. Though some AGN studies find a correlation with major mergers (Koss et al. 2010; Ellison et al. 2011), AGNs found with other selection techniques and at different redshift ranges do not show a higher rate of merging than field galaxies (Cisternas et al. 2011b; Kocevski et al. 2012). Instead of mergers bringing in gas, some systems probably are undergoing secular evolution, accreting their gas directly from the cold intergalactic medium. Bar and spiral disk instabilities are capable of dissipating sufficient angular momentum from this gas to fuel black hole growth (Hopkins \& Quataert 2010a, 2011). The dividing line between these two processes is not yet well understood; here we focus on understanding the black hole growth due to major mergers.

Nearby (Ultra-)Luminous InfraRed Galaxies ((U)LIRGs; Sanders \& Mirabel 1996) are an excellent sample with which to study such gas-rich mergers. These galaxies have infrared luminosities upwards of $10^{11} L_{\odot} \quad\left(10^{12} L_{\odot}\right.$ for ULIRGs $)$, generally caused by a starburst and/or an AGN heating up dust. Their infrared luminosities correlate with merger rate, star formation rate, and AGN fraction (Ellison et al. 2013). In fact, in the local universe, such strong infrared activity is almost exclusively triggered by major mergers: Veilleux et al. (2002) showed that in a complete sample of IRAS ULIRGs, 117 out of 118 galaxies are in the midst of strong tidal interactions.

In a companion paper (Medling et al. 2014), we have studied the kinematics of both gas and stars in the inner kiloparsecs of a sample of (U)LIRGs, finding that nuclear disks on scales of a few tens to hundreds of parsecs are common. In two other papers (Medling et al. 2011; U et al. 2013), we demonstrated a technique that uses high spatial resolution integral field spectroscopy to measure black hole masses. With kinematic maps that resolve inside the sphere of influence of a black hole, the complex and unrelaxed large-scale dynamics are less important, and black hole masses can be measured to within a factor of a few. We note that this technique measures the unresolved central mass, which includes both the black hole and its accretion disk, and in some cases may also include a reservoir of gas feeding the accretion disk. In U et al. (2013), the black hole mass in Mrk $273 \mathrm{~N}$ measured with this technique was consistent with the measurement made by $\mathrm{OH}$ maser kinematics (Klöckner and Baan 2004).

Using a robust technique such as this to measure black hole masses in these gas-rich mergers is important because more traditional methods of black hole mass measurements rely on assumptions that are not valid in the case of galaxy mergers. Three-integral orbital superposition models (as in Gültekin et al. 2009; Siopis et al. 2009) are able to use large-field kinematics to separate different components to the mass profile of the galaxy; however, this approach requires a dynamically relaxed system and is used therefore in isolated galaxies. Another successful black hole mass measurement technique is reverberation mapping (e.g., Denney et al. 2009, and references therein), which measures the time lag between flux variations of the continuum and the lines in the broad line region. Since the cores of (U)LIRGs are so dusty, the broad line regions are too obscured to view.

Obtaining data at spatial resolutions sufficiently high to resolve inside the sphere of influence of a supermassive black hole at the typical redshifts of local (U)LIRGs $(z \lesssim 0.1)$ requires adaptive optics systems, which are becoming available at an increasing number of ground-based observatories. Though the Hubble Space Telescope (HST) has excellent resolution in the visible bands, its relatively small mirror size limits the resolution at the longer wavelengths $(>2 \mu \mathrm{m})$ necessary to look through the dust in these galactic nuclei; even at the longest wavelength available to the Wide Field Camera 3 ( $H$-band), these nuclei are still sometimes obscured. In order to achieve high spatial resolution in $K$-band, large ground-based telescopes have employed adaptive optics systems which measure turbulence in the Earth's atmosphere and use a deformable mirror to correct for the resulting distortions. These distortion measurements require references, either a natural guide star (NGS AO) or a laser guide star plus a fainter natural "tip-tilt" star (LGS AO). The addition of laser guide star adaptive optics has increased the area of the sky observable with this technique.

Throughout this paper we have adopted a cosmology of $H_{0}=70 \mathrm{~km} \mathrm{~s}^{-1} \mathrm{Mpc}^{-1}, \Omega_{m}=0.28$, and $\Omega_{\Lambda}=0.72$ (Hinshaw et al. 2009). In Section 2 we present our data and reduction techniques. In Section 3 we briefly describe the kinematic fitting techniques demonstrated in Medling et al. (2011) and U et al. (2013). In Section 4 we present the black hole masses measured from several tracers and in Section 5 compare them to black hole scaling relations. Section 6 contains our conclusions.

\section{OBSERVATIONS}

\subsection{The Sample}

We have selected nine gas-rich merging galaxies in which to measure the black hole masses using gas and stellar kinematics. These galaxies represent the subset of merging galaxies presented in Medling et al. (2014) for which high quality 
Table 1

Details of Observations

\begin{tabular}{|c|c|c|c|c|c|c|}
\hline Galaxy Name & Redshift & $\begin{array}{l}\text { Pixel Scale } \\
\left(\mathrm{pc} / 0 .^{\prime \prime} 035\right)\end{array}$ & UT Date(s) & Filter & $\begin{array}{c}\text { Exp Time on } \\
\text { Target (minutes) }\end{array}$ & $\begin{array}{c}\text { Exp Time on } \\
\text { Sky (minutes) }\end{array}$ \\
\hline CGCG436-030 & 0.0315 & 21.8 & 2012 Sep 30 & $\mathrm{Kn} 5$ & 30 & 5 \\
\hline IRASF01364-1042 & 0.0493 & 33.6 & 2012 Oct 01 & $\mathrm{Kbb}$ & 20 & 10 \\
\hline MCG+08-11-002 & 0.0195 & 13.9 & 2012 Jan 02 & $\mathrm{Kbb}$ & 90 & 50 \\
\hline NGC 2623 & 0.0196 & 13.9 & 2010 Mar 2004, 2010 Mar 05 & Kn5 & 100 & 50 \\
\hline UGC $5101^{\mathrm{a}}$ & 0.0413 & 27.0 & 2010 Mar 2004, 2010 Mar 05 & Kn5 & 80 & 50 \\
\hline NGC $6240 \mathrm{~S}^{\mathrm{c}}$ & 0.0244 & 17.2 & 2007 Apr 21 & Kn5 & 20 & 10 \\
\hline IRASF17207-0014 ${ }^{\mathrm{a}}$ & 0.0462 & 29.8 & 2011 May 23, 2011 May 24 & $\mathrm{Hn} 4$ & 40 & 20 \\
\hline
\end{tabular}

Notes.

a Originally presented in Medling et al. (2014).

${ }^{\mathrm{b}}$ Originally presented in U et al. (2013).

${ }^{c}$ Originally presented in Medling et al. (2011).

kinematics data exist. The parent sample was drawn from the Great Observatories All-sky LIRG Survey (GOALS; Armus et al. 2009), which targeted about two hundred of the brightest infrared galaxies in the sky $\left(\log \left(L_{\mathrm{IR}} / L_{\odot}\right)>11.0\right)$. Our targets were selected from that sample for the following criteria: available archival $B$ - and $I$-band imaging from the $H S T$ Advanced Camera for Surveys; visible double or extended cores or clumps in the central five arcseconds (within our field of view (FOV)); large-scale morphology consistent with that of a major merger; observable from Keck Observatory and with an appropriate guide star $\left(\leqslant 18\right.$ th magnitude in $R, \leqslant 60^{\prime \prime}$ separation).

Our sample is comprised of (U)LIRGs involved in major gas-rich galaxy mergers, with a bias toward later stages of merging. These galaxies are nearby, with redshifts $\leqslant 0.05$, which is required to achieve spatial resolutions of $\lesssim 30$ pc per pixel $(\lesssim 50$ pc per resolution element $)$.

\subsection{The Data}

We obtained near-infrared integral field spectroscopy of the central kiloparsec of nine merging galaxies with OSIRIS, the OH-Suppressing InfraRed Imaging Spectrograph (Larkin et al. 2006) on the W. M. Keck I (after August 2012) and II (before August 2012) 10 meter telescopes. All data presented here uses the $0.035 \operatorname{arcsec}^{\text {spaxel }}{ }^{-1}$ plate scale. This high spatial resolution is enabled by the Keck Observatory LGS AO system (Wizinowich et al. 2000; van Dam et al. 2004, 2006; Wizinowich et al. 2006).

Our OSIRIS observations were typically comprised of observing sets of object-sky-object; each exposure was ten minutes ( 5 minutes for Keck I observations). Observations were taken either in the broadband $K$ filter (Kbb: $1.965-2.381 \mu \mathrm{m})$ or in a narrow band targeting specific lines (Hn4: 1.652-1.737 $\mu \mathrm{m}$; Kn5: $2.292-2.408 \mu \mathrm{m})$. When possible, the primary target lines were the $\mathrm{CO}(2-0)$ and (3-1) bandheads at 2.293 and $2.323 \mu \mathrm{m}$ in $K$, which trace the kinematics of young stars. When available, we compare the stellar kinematics to those of various emission lines: [Fe II] $1.644 \mu \mathrm{m}, \mathrm{Br} \gamma 2.16 \mu \mathrm{m}, \mathrm{H}_{2} 2.12 \mu \mathrm{m}$. For some galaxies, stellar kinematics were not available because the $\mathrm{CO}$ bandheads are redshifted out of $K$, and we rely on the aforementioned emission lines for this analysis. Total exposure times, observed filters, and parameters of the observed galaxies are listed in Table 1. Some of these data were presented in previous papers (Medling et al. 2011; U et al. 2013; Medling et al. 2014); these are indicated in Table 1 as well.

We reduced our Keck II observations using the OSIRIS Data Reduction Pipeline ${ }^{9}$ version 2.3 (using the updated OSIRIS wavelength solution for data taken after October 2009) and our Keck I observations using version 3. This pipeline includes modules to subtract sky frames, adjust channel levels, remove crosstalk, identify glitches, clean cosmic rays, extract a spectrum for each spatial pixel, assemble the spectra into a data cube, correct for atmospheric dispersion, perform telluric corrections, and mosaic frames together. For some cases we utilized the Scaled Sky Subtraction module based on the technique outlined in Davies (2007), which scales the thermal continuum and $\mathrm{OH}$ line groups separately to provide optimal sky subtraction; we modified the module to include a smoother subtraction of the thermal continuum, as described in Medling et al. 2014.

We imaged the tip-tilt stars for each galaxy during the course of the observations in order to obtain an estimate of the pointspread function (PSF). A Moffat function was fit to each tip-tilt star and then broadened according to the distance between the target and the tip-tilt star. This broadening accounts for the fact that the laser spot is not at infinity; therefore it only probes most of the turbulence that affects the target. As a result, the further a target is from its tip-tilt star, the worse its PSF will be. The isokinetic angle for the Keck II AO system, at which the Strehl ratio is reduced to $37 \%$ of its peak value, is approximately 75 arcseconds. We also use this an estimate of the Keck I AO system performance, which has not yet been characterized.

\section{LINE FITTING}

We measured the kinematics following the methods presented in detail in Medling et al. (2011, for stellar kinematics) and $\mathrm{U}$ et al. (2013, for emission line kinematics), and reviewed again in Medling et al. (2014). We briefly describe these techniques here.

For each measurement, we first calculate the signal-to-noise ratio $(\mathrm{S} / \mathrm{N})$ in each pixel and bin them using optimal Voronoi tesselations (Cappellari \& Copin 2003) in order to require a certain $\mathrm{S} / \mathrm{N}$ for reliable measurements. We used thresholds of

\footnotetext{
9 Available at http://irlab.astro.ucla.edu/osiris/pipeline.html.
} 
20 and 3 per resolution element for stellar kinematics and emission line kinematics, respectively. Once binned, stellar kinematics were fit using the penalized pixel fitting routine ${ }^{10}$ (Cappellari \& Emsellem 2004) and using $K$-band templates of late-type giants and supergiants from GNIRS (Winge et al. 2009). Emission lines were fit with Gaussian profiles to determine the flux, velocity, and velocity dispersion in each bin. For emission lines where multiple lines exist in a specific band (the five $\mathrm{H}_{2}$ transitions in the $K$-band, or $\operatorname{Br} \delta$ and $\operatorname{Br} \gamma$ ), those lines were fit simultaneously, requiring that the velocity and velocity dispersion be consistent between lines.

\section{BLACK HOLE MASSES}

Our black hole mass measurement techniques have been demonstrated in two prior papers. In Medling et al. (2011), we place limits on the mass of the black hole in the south nucleus of NGC 6240 using 2D stellar kinematic maps and two methods. We calculate the lower limit to the mass by assuming the stars lie in a thin Keplerian disk, and fit a density profile including a black hole and a smoothly varying spheroidal mass component: $\rho(r)=M_{\mathrm{BH}}+\rho_{0} r^{-\gamma}$. Because of the high spatial resolution of our data, we probe radii small enough that the first term dominates over the second. The measured black hole mass here is a lower limit because this method ignores velocity dispersion (assuming that all measured velocity dispersion is due to material along the line of sight, not intrinsic to the disk). Any intrinsic velocity dispersion in the disk will be indicative of extra mass in the black hole. In the second scenario, we obtain an upper limit on the black hole mass by assuming the opposite: that all measured velocity dispersion is intrinsic to the disk. Because the system is unrelaxed, there is unvirialized material along the line of sight increasing the velocity dispersion but not knowing about the central black hole mass. To find this limit, we use Jeans Axisymmetric Mass models (JAM; Cappellari 2008). We use both methods here for all galaxies with measured stellar kinematics, except for the north black hole in NGC 6240. This nucleus contains patchy dust that prevents a consistent light profile from being produced by the JAM models; in order to use this measurement technique on that black hole, equivalently high resolution imaging at a longer wavelength ( $L$-band or beyond) is required.

In $\mathrm{U}$ et al. (2013), we measure the mass of the black hole in the north nucleus of Mrk 273 in a similar manner. Since no stellar kinematics were available, the JAM models were not feasible. When only gas kinematics are available, we model the gas as a thin Keplerian disk and proceed as in the first case above. The black hole mass measured for this galaxy agrees well with the measurement made using the kinematics of an OH maser (Klöckner and Baan 2004), and therefore provides independent justification for this technique. We note that the thin Keplerian disk approximation provides reasonable $M_{\mathrm{BH}}$ agreement to the maser measurement even though the disks analyzed in Medling et al. (2014) are thick rather than thin, with $v / \sigma \sim 1-4$. We note that this thickness may cause our black hole mass measurements to underestimate the true black hole mass by up to $\sim 25 \%$.

With this approach, we are measuring the unresolved central mass within $\sim 25$ parsecs. This is likely dominated by the black hole, and we therefore refer to it as such. However, this mass includes the accretion disk of the black hole and may include a

\footnotetext{
10 Available at http://astro.physics.ox.ac.uk/m̃xc/idl.
}

reservoir of gas feeding the accretion disk. If the accretion disk is fueled smoothly by the nuclear disk, this mass will be accounted for by the radial profile; we cannot account for a pile-up of gas below our resolution limit. The possible implications for this will be discussed further in Section 5.

In each case, errors on the black hole mass measurement were obtained using a Monte Carlo approach. We created a model datacube and added random noise appropriate to the S/N of each spectrum and then refit the black hole mass for 100 iterations. The width of the resulting mass distribution is the error on the measurement.

We have performed the above analysis for nine additional black holes, listed in Table 1, and include the results in Table 2 and the fits in Appendix A. As in previous papers, these methods produce kinematic models in reasonable agreement with observations for most cases. The ability to measure black hole masses using different tracers, and additionally to use bracketing assumptions about velocity dispersion for stellar models, improves the robustness of these results.

The numerical results from our black hole mass measurements are listed in Table 2, along with parameters relevant to black hole scaling relations, pulled from the literature: the stellar velocity dispersion of the bulge, the stellar mass of the bulge, and the total stellar mass of the galaxy. Though consistent treatment of such parameters when drawing from different literature sources is difficult, care was taken to select measurements most appropriate to the scaling relation. The bulge luminosities and total stellar masses were taken from the GOALS papers of Haan et al. (2011) and U et al. (2012), respectively. Haan et al. (2011) measured the bulge luminosities using GALFIT (Peng et al. 2002, 2010) to decompose $H$ band NICMOS images into multiple Sérsic components. These galaxies are morphologically complex, which can produce an increased uncertainty in bulge fit parameters; thus, the errors in bulge luminosities reported by Haan et al. (2011) and reproduced in Table 2 may be larger than the equivalent measurements from Hubble imaging of isolated galaxies. It is worth considering that we use the formal statistical errors produced by GALFIT; the analysis of Häussler et al. (2007) has shown that these underestimate the true errors, which can contain contributions from profile mismatch and nearby neighbors, both relevant to our sample. However, Häussler et al. (2007) also find that GALFIT shows no systematic offset to fitting bulge luminosities of bright objects and that it is able to appropriately handle contamination by neighbors when fit simultaneously (as was done in Haan et al. 2011). Still, for a more complete understanding of the errors associated with each bulge luminosity measurement, we refer the readers to the models and residuals for each system of Haan et al. (2011), published online in Figure Set 7. Our total stellar masses were measured from SEDs using photometry masks (U et al. 2012) designed to incorporate all of the light from the galaxy. We are thus not underestimating the total stellar mass, as can happen with fixed-aperture photometry, and we avoid potential biases related to varying host galaxy effective radius (Hopkins et al. 2007a, 2007b; Hopkins et al. 2009; Beifiori et al. 2012). The GOALS survey papers allow us to be confident that the bulge luminosities and total stellar masses were measured consistently across our sample. However, no such survey paper exists currently to measure the stellar velocity dispersions of the bulges. Instead, we selected the stellar velocity dispersion measurement from the aperture that most closely aligned with 
Table 2

Measured Black Hole Masses

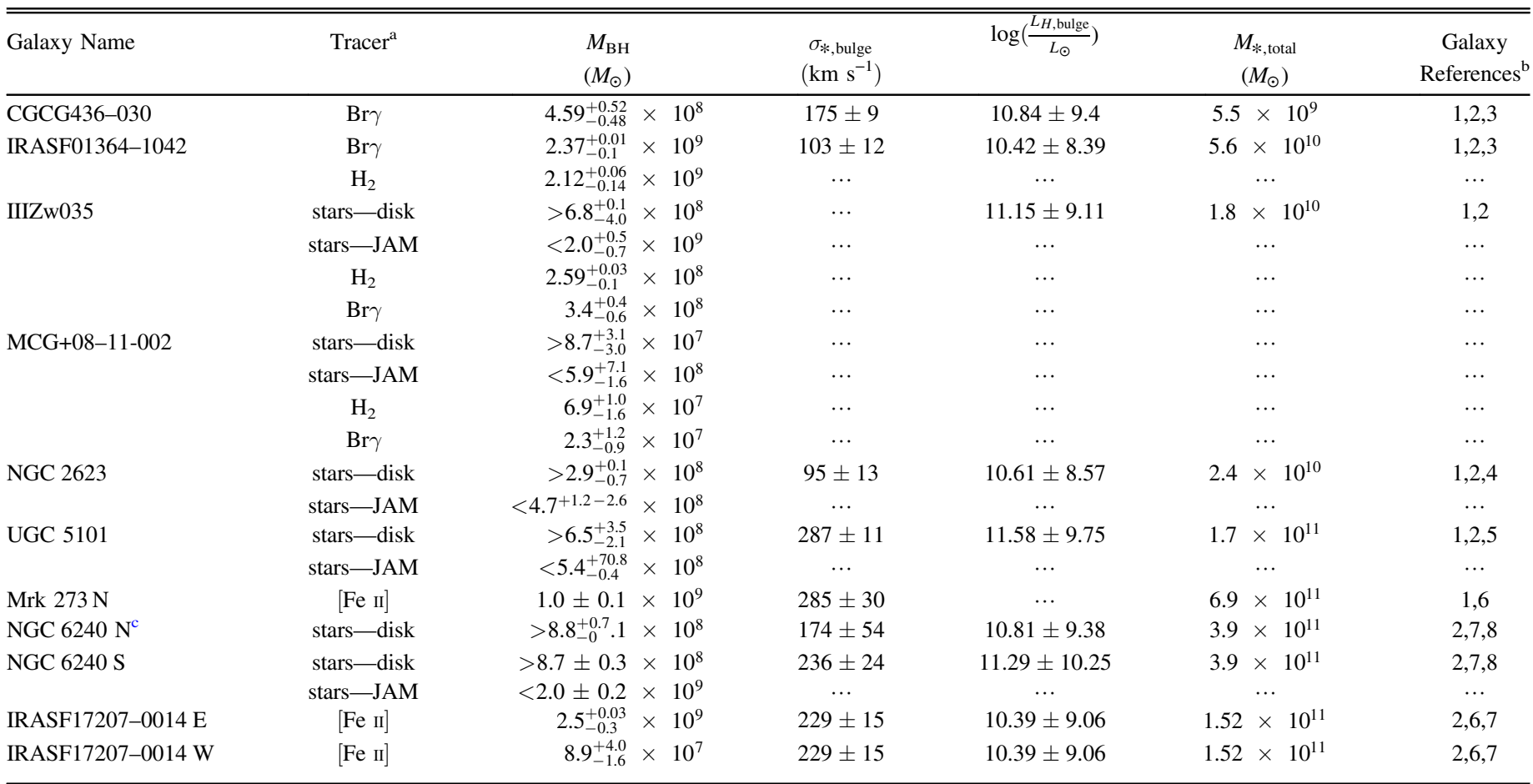

Notes.

${ }^{a}$ All measurements with gas tracers use the thin disk method, those with stellar tracers use the disk or JAM method as marked.

b Galaxy parameters in columns 4-6 taken from the following references: (1) U et al. (2012), (2) Haan et al. (2011), (3) Tremonti et al. (2004), (4) Shier et al. (1996), (5) Rothberg \& Joseph (2006), (6) Dasyra et al. (2006), (7) Howell et al. (2010), (8) Tecza et al. (2000).

${ }^{c}$ Due to extensive and patchy dust coverage, a JAM model could not be completed for NGC $6240 \mathrm{~N}$ without additional longer-wavelength $(L$-band or beyond) imaging data.

the size of the bulge. Unfortunately, no bulge information for MCG+08-11-002 is available in the literature. Although it cannot be placed on scaling relations at this time, we include our black hole mass measurements for completeness.

\section{MERGERS AND BLACK HOLE GROWTH}

\subsection{Placing Black Holes on Scaling Relations}

Using our measured black hole masses and host galaxy parameters from the literature, all listed in Table 2, we place the galaxies on several black hole scaling relations.

\subsubsection{The $M_{\mathrm{BH}}-\sigma_{*}$ Relation}

In Figure 2, we show the $M_{\mathrm{BH}}-\sigma_{*}$ relation from McConnell $\&$ Ma (2013) with our points overlaid.

The black holes in our sample lie either within the scatter of the relation or above it. Although a particular galaxy may fall within the scatter of the relation, we want instead to consider whether our sample as a whole is behaving as predicted by the $M_{\mathrm{BH}}-\sigma_{*}$ relation. We calculate the distances from the $M_{\mathrm{BH}}-\sigma_{*}$ relation for both our data set and the reference population, and then perform a two-sided Kolmogorov-Smirnoff test on the two populations. We find a $p$-value of 0.003 , indicating that the chance of these two being drawn from the same population is less than $1 \%$. To mitigate the possible effects of outliers in our small sample, we use the balanced

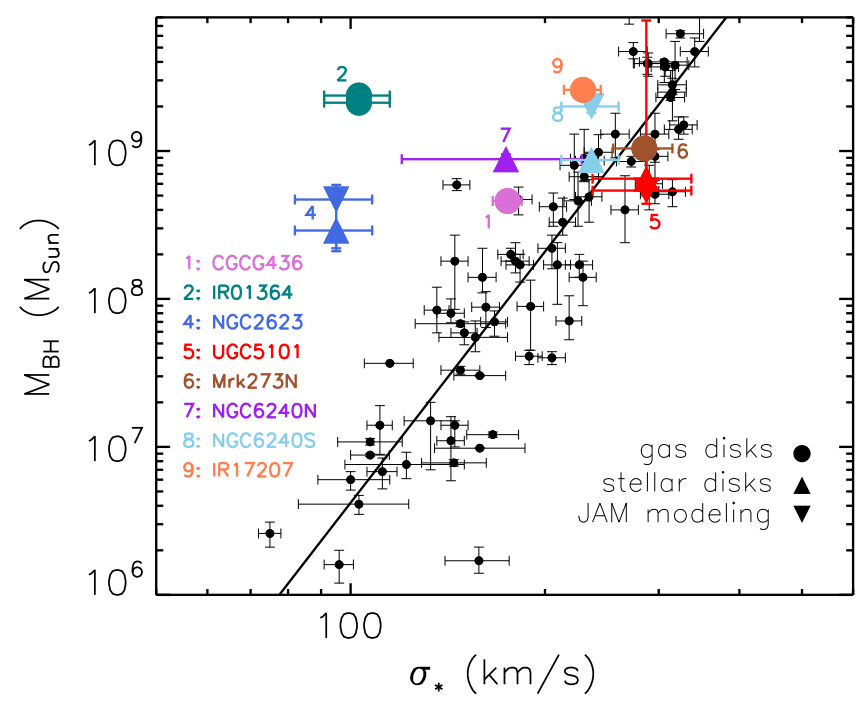

Figure 2. The $M_{\mathrm{BH}}-\sigma_{*}$ relation for isolated galaxies from McConnell \& Ma (2013) (black data and best-fit line: $\log \left(M_{\mathrm{BH}} / M_{\odot}\right)=8.32+5.64 \log \left(\sigma_{*} / 200\right.$ $\mathrm{km} \mathrm{s}^{-1}$ ) with our merging galaxies marked in large colored points. Symbols indicate which method was used for that mass determination: gas disk modeling in circles, stellar disk modeling in upward triangles, and JAM modeling in downward triangles. Stellar disk models represent lower limits to the black hole mass, while JAM models represent upper limits; see text for details. Mrk 273 N's point (6; brown) is the mass measurement for only the north black hole, though there is a second black hole in the system (see $\mathrm{U}$ et al. 2013). 
bootstrap method ${ }^{11}$ to estimate the average offset from the relation. This reveals an average offset of $0.15 \pm 0.06$ in plot units; we thus consider this offset a $2.6 \sigma$ result.

Our sample of black holes are thus, on average, above the relation, suggesting with moderate significance that black holes may grow in mergers before the bulges are virialized. Measuring the velocity dispersion of stars in the bulge can be difficult to define when two bulges are in the process of merging. This variation in measured velocity dispersion (both due to the process of merging and due to geometric variations based on the line of sight (LOS)) has been quantified in the hydrodynamical galaxy merger simulations of Stickley \& Canalizo (2014). Their analysis shows a maximum predicted mismeasurement in velocity dispersion of approximately $50 \%$, which is insufficient to bring all our systems onto the relation.

\subsubsection{The $M_{\mathrm{BH}}-L_{H, \text { bulge }}$ Relation}

In Figure 3, we show the $M_{\mathrm{BH}}-L_{H, \text { bulge }}$ relation. The background galaxies were plotted using $L_{H, \text { bulge }}$ from Marconi \& Hunt (2003), with updated black hole masses from McConnell \& Ma (2013). We have updated the best-fit line with these numbers, producing the relation:

$$
\log \left(\frac{M_{\mathrm{BH}}}{M_{\odot}}\right)=8.22+1.06 \log \left(\frac{L_{H, \text { bulge }}}{10^{10.8} L_{\odot}}\right) .
$$

The black holes from our sample fall either within the scatter or above the $M_{\mathrm{BH}}-L_{H \text {,bulge }}$ relation. As with the $M_{\mathrm{BH}}-\sigma_{*}$ relation, we perform a two-sided Kolmogorov-Smirnoff test to determine if our sample as a whole falls significantly above the relation; we find a $p$-value of 0.018 , indicating that these black hole masses are unlikely to be pulled from the sample distribution as the reference sample. We also calculate the mean offset using the balanced bootstrap method, $0.47 \pm 0.14$ in plot units, showing an offset with $\sim 3.4 \sigma$ significance.

We note two possible calibration effects when considering our bulge luminosity measurements. First, although Marconi \& Hunt (2003) did not correct their bulge luminosities for internal extinction, we consider the effects such a correction would have on our sample because our galaxies are quite dusty. Several of our galaxies have $J H K$ imaging published by Scoville et al. (2000), enabling a direct estimate of typical extinction over the central few kiloparsecs. These calculations estimate that the intrinsic luminosity of the bulges in our sample may be $\sim 10 \%$ higher. This correction (indicated by red arrow in the top left of Figure 3) is smaller than the size of our points and can be safely ignored. Second, we investigate whether the varying bulge Sérsic indices of the fits in Haan et al. (2011) may be biasing our luminosity estimates. When fixing $n$ to 4 , the bulge luminosities of several of our galaxies change significantly, some higher and some lower (S. Haan 2015 , private communication). The net effect with this change is less scatter between our galaxies: a smaller but more statistically significant offset. We conclude that, while the Sérsic index fit may have a strong effect on an individual system's predicted black hole mass when using the $M_{\mathrm{BH}}-L_{H \text {,bulge }}$ relation, the offset in our data is not caused by

\footnotetext{
${ }^{11}$ Balanced bootstrap resampling is similar to ordinary bootstrap resampling but requires that each observed value appears with equal frequency in the resampled data (e.g., Gleason 1988). We used the IDL routine BBOOTSTRAP, available at http://astro.washington.edu/docs/idl/cgi-bin/getpro/ library 14.htmlXXBBOOTSTRAP.
}

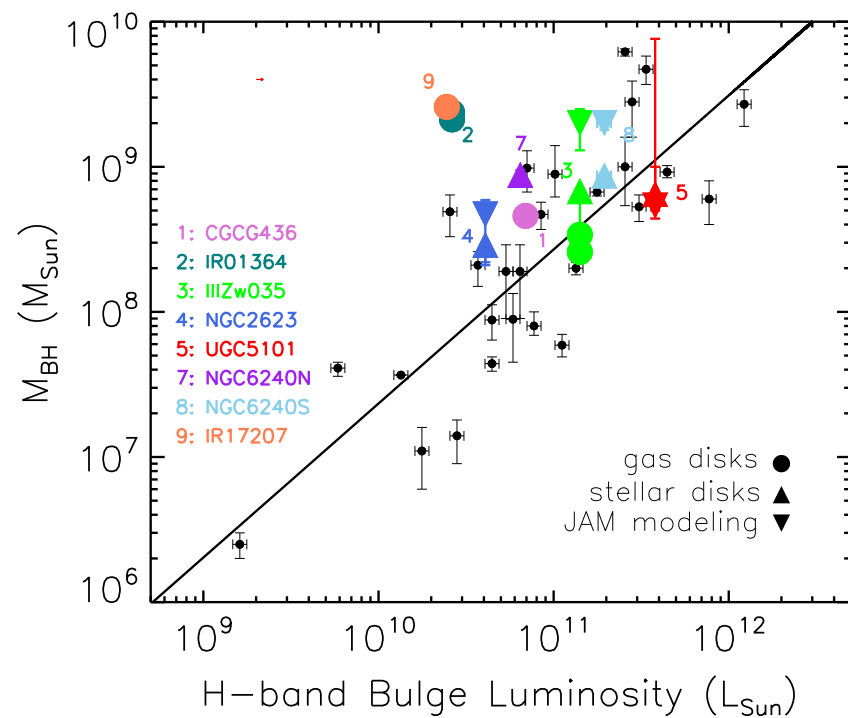

Figure 3. The $M_{\mathrm{BH}}-L_{H, \text { bulge }}$ relation. The black points have luminosities taken from Marconi \& Hunt (2003) and updated black hole masses from McConnell \& Ma (2013). The solid line is a $\log -\log$ fit to the updated data: $\log \left(\frac{M_{\mathrm{BH}}}{M_{\odot}}\right)=8.22+1.06 \log \left(\frac{L_{H, \text { bulge }}}{10^{10.8} L_{\odot}}\right)$. Galaxies presented here are indicated by large colored points, with parameters listed in Table 2. The bulge luminosities were not corrected for extinction; the arrow in the top left shows the typical magnitude of such a correction $(10 \%)$. Symbols indicate which method was used for that mass determination: gas disk modeling in circles, stellar disk modeling in upward triangles, and JAM modeling in downward triangles. Stellar disk models represent lower limits to the black hole mass, while JAM models represent upper limits; see text for details.

the fitting technique of these bulge luminosities. We also note that the two galaxies which fall farthest above the relation, IRASF01364-1042 and IRASF17207-0014, do not show atypically large residuals in the GALFIT bulge fits (indeed, the residuals of the former appear smaller than average for this sample), which suggests that their offset is not due to errors in the bulge luminosities of Haan et al. (2011).

Again, our sample of merging galaxies falls above this relation, suggesting that either stars have not been forming in the bulge as quickly as the black holes have been growing, or that a large mass of stars is still strewn about in tidal tails and unrelaxed features that will eventually become part of the bulge. As the $H$-band traces older populations of stars, it may be that some stars have recently formed in the bulge, but that this measurement misses them. Although $M_{\mathrm{BH}}-L_{\text {bulge }}$ relations do exist for bluer colors, we are unfortunately unable to utilize them for this sample because the large amounts of dust obscure the galaxy cores, making bulge luminosity measurements in the optical highly uncertain. We have compared our bulge luminosities to those fit by Kim et al. (2013) in the I-band, and found that (for typical mass-to-light ratios) our $H$-band bulges are more massive. This simply confirms that dust is a bigger problem even in the $I$-band than missing stellar populations are in the $H$-band for this sample.

$$
\text { 5.1.3. The } M_{\mathrm{BH}}-M_{* \text {,total }} \text { Relation }
$$

In Figure 4, we plot $M_{\mathrm{BH}}-M_{* \text {,total }}$ using points from Bennert et al. (2011) and Cisternas et al. 2011a. These authors suggest that higher redshift galaxies sit on the local $M_{\mathrm{BH}}-M_{* \text {,bulge }}$ relation if you include total stellar mass instead of only bulge mass. In this way, they attribute evolution in the black hole scaling relation to the evolution of the fraction of stellar mass in 


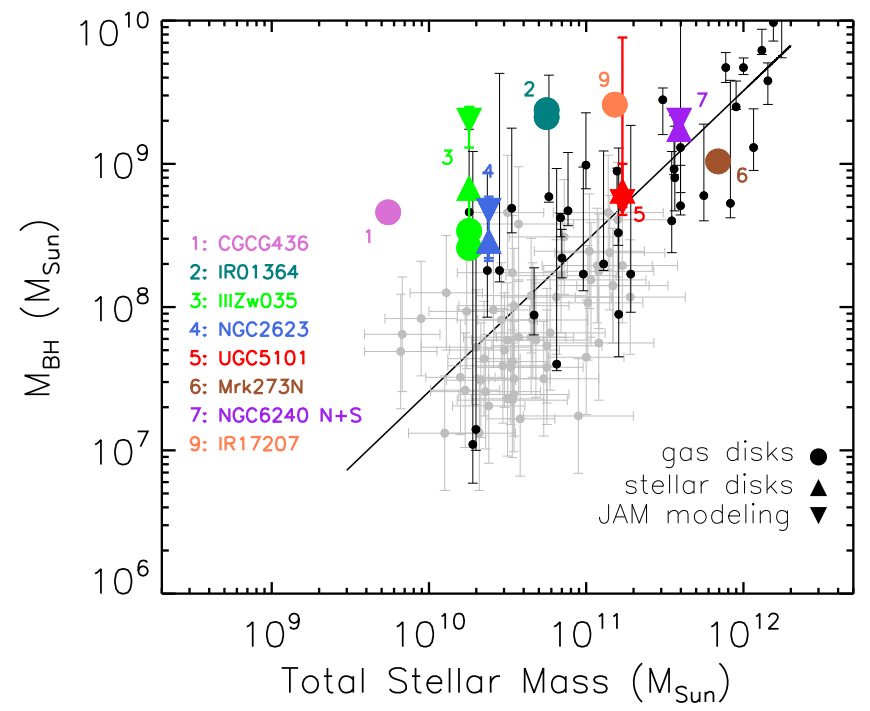

Figure 4. The $M_{\mathrm{BH}}-M_{*}$ relation. The gray points have luminosities and total stellar masses from Bennert et al. (2011) and Cisternas et al. (2011a), who suggest that higher redshift galaxies sit on the local $M_{\mathrm{BH}}-M_{*, \text { bulge }}$ relation if you include total stellar mass instead of only bulge mass. In this way, they attribute evolution in the black hole scaling relation to the evolution of the bulge/disk fraction with redshift. We include also black points for local

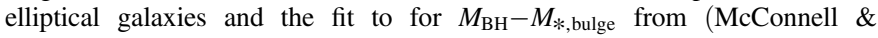
Ma $2013, \log \left(M_{\mathrm{BH}} / M_{\odot}\right)=8.46+1.05 \log \left(M_{*} / 10^{11} M_{\odot}\right)$. Merging galaxies from this work are indicated by large colored points, with parameters listed in Table 2. Symbols indicate which method was used for that mass determination: gas disk modeling in circles, stellar disk modeling in upward triangles, and JAM modeling in downward triangles. Stellar disk models represent lower limits to the black hole mass, while JAM models represent upper limits; see text for details. NGC6240's two nuclei were summed together and appear as one point (7; dark purple) on this plot, paired with the entire system's total stellar mass. Mrk 273 N's point (6; brown) is the mass measurement for only one black hole, though there is a second in the system (see U et al. 2013).

the bulge. We include as a solid line the updated fit to the

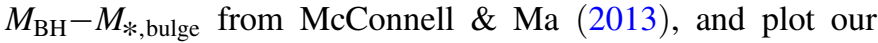
black hole masses in the same colored points as in Figures 2 and 3 .

To determine if this sample of systems is in agreement with the $M_{\mathrm{BH}}-M_{* \text {,total }}$ relation, we perform a similar two-sided Kolmogorov-Smirnoff statistical test as above. We find that, compared to the local elliptical galaxies of McConnell \& Ma (2013), the $p$-value obtained is only 0.01 , less significant than above. When comparing to the higher-redshift samples of total stellar mass, we obtain a $p$-value of 0.0008 , and to the combined sample, a $p$-value of 0.004 . The balanced bootstrap method finds an offset from the McConnell \& Ma relation of $0.46 \pm 0.14$ in plot units, similar to the $M_{\mathrm{BH}}-L_{\text {bulge }}$ relation.

We thus conclude that our sample of galaxies on average lies above this scaling relation as well. We also note that the one point that falls below the relation, the brown circle Mrk $273 \mathrm{~N}$ (labeled 6), represents the mass in only one out of two black holes and is therefore artificially low. Because this relation takes into account the entirety of stellar mass, the fact that many of our points fall above the relation suggests that star formation as a whole is not outpacing black hole growth during these mergers.

\subsubsection{Overall Significance of Scaling Relation Offset}

In the previous sections, we have compared our galaxies with the $M_{\mathrm{BH}}-\sigma_{*}$ and $M_{\mathrm{BH}}-M_{*, \text { bulge }}$ relations from McConnell
\& Ma (2013). We have also compared them to those from Kormendy \& Ho (2013), and find that our offset remains.

Two galaxies appear to drive the offset in the $M_{\mathrm{BH}}-\sigma_{*}$ relation: IRASF01364-1042 and NGC 2623. Although we note that these are not outliers in a statistical sense, we must be certain that they are not unfairly biasing our results. Because our sample for each scaling relation has only eight points, throwing out these two galaxies has a significant effect in all cases through reducing the sample size. We note, however: when removing the two galaxies from the $M_{\mathrm{BH}}-\sigma_{*}$ relation, the measured offset decreases quite a bit, while the uncertainty in the offset also increases (producing only a $1.9 \sigma$ result). For the other two relations, the measured offset decreases only slightly, and the main effect is an increase in uncertainty (so the significance still decreases from $3.3 \sigma$ to $2.3 \sigma$ ). This demonstrates that the offsets are not driven by these two galaxies in the $M_{\mathrm{BH}}-L_{\text {bulge }}$ and $M_{\mathrm{BH}}-M_{* \text {,total }}$ relations.

Due to the small sizes of our samples, the appropriate way to deal with potential outliers is to use the balanced bootstrap test, which we have done. The balanced bootstrap performs hundreds of resamplings in order to estimate the significance of a result, giving equal weight to all measurements. (Non"balanced" bootstrapping may resample some points more than others, which could cause a bias when one or more potential outliers exist. By balancing the bootstrapping, we force the equal treatment of all measurements; this prevents a single point (or two) from biasing the results.) By doing so, we are able to handle outliers (or near-outliers) without diminishing our sample size.

The black holes in this sample of gas-rich major mergers consistently fall on or above the scaling relations for passive galaxies with stellar velocity dispersion, bulge luminosity, and total stellar mass. Although a number of our galaxies lie within the scatter of these scaling relations, our statistical tests show they are significantly more likely to fall on the upper side of the scatter than randomly distributed about the relations. Though the offset from the $M_{\mathrm{BH}}-\sigma_{*}$ relation is only a $2.6 \sigma$ result, the agreement with the two other significant relations lends it confidence. Taken together, these results show that increased black hole growth in these mergers has already begun and may be proceeding more rapidly than growth of the host galaxy.

Although we have a relatively small sample of black holes, it is striking that we already see a significant offset. As more black hole masses are measured in gas-rich mergers, the larger sample will provide a more accurate picture of how offset such systems are from black hole scaling relations.

We caution the reader against considering the distance from a scaling relation as a direct proxy for merger stage. This is clearly evident when comparing where a system falls on one scaling relation versus another. For example, IRASF17207-0014 falls closer to the $M_{\mathrm{BH}}-\sigma_{*}$ relation than to the $M_{\mathrm{BH}}-L_{H \text {,bulge }}$ relation; for NGC $6240 \mathrm{~S}$, the reverse is true. This discrepancy could be caused by at least two possible mechanisms: (1) the host galaxy parameters trace different aspects of galaxy growth, which may evolve differently. For example, the bulge velocity dispersion traces a convolution of mass growth with the virialization timescale whereas the bulge luminosity evolution may rely less stringently on the dynamical relaxation time. (2) As discussed above, the simulations of Stickley \& Canalizo (2014) show that the measured stellar velocity dispersion of similar mergers oscillates during the course of the merger. Analogous simulations would have to be 
Table 3

Black Hole Growth Timescales

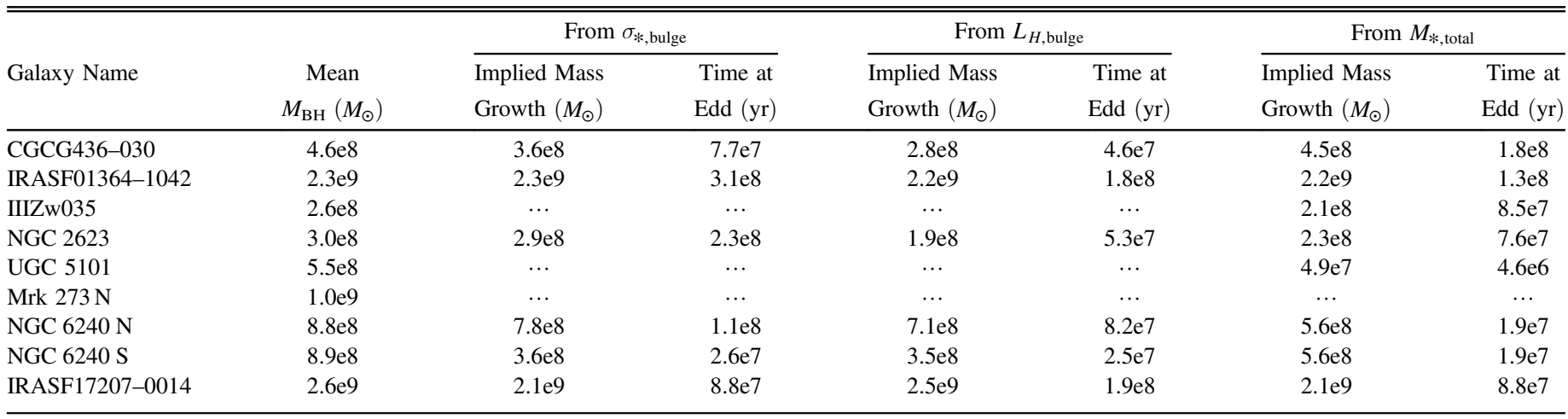

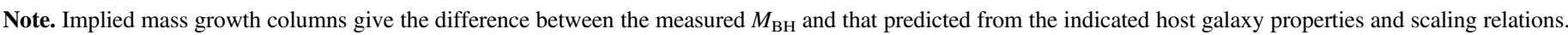

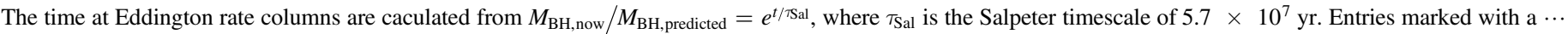
indicate either systems that do not have measured host galaxy properties or that lie on or below scaling relations and therefore require zero growth.

performed for bulge luminosity and total stellar mass measurements to determine if they ought to oscillate as well. Even if all three quantities were expected to oscillate during a merger, it seems contrived to require that they oscillate in lockstep. To determine precisely how galaxies evolve along a scaling relation (and if they evolve the same way along each scaling relation), a larger sample of systems is required. By binning over merger stage, the range of distances from scaling relations can be seen, and key parameters affecting this can be identified. We leave this analysis for future studies.

\subsection{Interpreting Overmassive Black Holes}

In this section, we discuss several plausibility arguments related to the above results. We consider the growth timescales and the available fuel for both black holes and the host galaxies.

\subsubsection{Has There Been Enough Time for these Black Holes to Grow?}

We posit that these systems followed black hole scaling relations before the merger began and that, since then, mass has been funneled to the center to enable black hole growth. For simplicity, we assume that each galaxy previously fell precisely on every scaling relation; of course, as each relation contains intrinsic scatter, this is unlikely. However, we have no reason to believe that these systems were systematically offset from scaling relations before the mergers began, and thus this simplification should average out in the order-of-magnitude calculations of this and the following sections.

With that assumption, we consider how long it would take for a black hole, growing at the Eddington rate, to reach this far beyond its original mass. In Table 3, we calculate this for our sample of galaxies. We see that the required times for these black holes to grow, while accreting at the Eddington rate, are a few tens to hundreds of millions of years. As this is less than or comparable to a merger timescale, it is reasonable that these systems could have lain on scaling relations before the merger, and risen only after the merger began. As black hole growth is known to be episodic, we note that these systems could often have been accreting at sub-Eddington rates for timescales less
Table 4

Star Formation Requirements

\begin{tabular}{lcccc}
\hline \hline & \multicolumn{3}{c}{ Time } \\
Galaxy Name & $\Delta M_{*, \text { total }}{ }^{\mathrm{a}}$ & $\begin{array}{c}\text { SFR } \\
\left(M_{\odot} \mathrm{yr}^{-1}\right)^{\mathrm{b}}\end{array}$ & $\begin{array}{c}\text { Required } \\
(\mathrm{yr})^{\mathrm{c}}\end{array}$ & $\begin{array}{c}\text { Available } \\
M_{\mathrm{H}_{2}}\left(M_{\odot}\right)^{\mathrm{d}}\end{array}$ \\
\hline CGCG436-030 & $1.5 \mathrm{e} 11$ & 85.87 & $1.7 \mathrm{e} 9$ & $\ldots$ \\
IRASF01364-1042 & $6.7 \mathrm{e} 11$ & 122.61 & $5.5 \mathrm{e} 9$ & $\ldots$ \\
IIIZw035 & $7.3 \mathrm{e} 10$ & $\ldots$ & $\ldots$ & $\ldots$ \\
NGC 2623 & $7.9 \mathrm{e} 10$ & 69.19 & $1.1 \mathrm{e} 9$ & $1.66 \mathrm{e} 9$ \\
UGC 5101 & $1.6 \mathrm{e} 10$ & 180.18 & $8.7 \mathrm{e} 7$ & $2.65 \mathrm{e} 9$ \\
Mrk 273 N & $\ldots$ & $\ldots$ & $\ldots$ & $\ldots$ \\
NGC 6240 N+S & $1.7 \mathrm{e} 11$ & 148.44 & $1.2 \mathrm{e} 9$ & $1.18 \mathrm{e} 10$ \\
IRASF17207-0014 & $6.6 \mathrm{e} 11$ & 501.22 & $1.3 \mathrm{e} 9$ & $7.13 \mathrm{e} 9$ \\
\hline
\end{tabular}

Notes.

${ }^{a} \Delta M_{*, \text { total }}$ gives the difference between the current $M_{*, \text { total }}$ (column 6 in Table 2) and that predicted from the current mean measured $M_{\mathrm{BH}}$ and the relevant scaling relation.

${ }^{\mathrm{b}}$ Star formation rates taken from Howell et al. (2010).

c Time required $=\Delta M_{* \text {,total }} / \mathrm{SFR}$.

${ }^{\mathrm{d}}$ From Wilson et al. (2008) when available.

e Mrk $273 \mathrm{~N}$ already falls on the scaling relation, and therefore no additional star formation is needed.

than those noted, and that the accretion times need not have been continuous.

The merger of one of these objects, NGC 2623, has been modeled by Privon et al. (2013) using H I kinematics from the Very Large Array. Their dynamical models indicate that this object is $2.2 \times 10^{8} \mathrm{yr}$ past first pericenter passage, within the range of growth timescales we see for this object in Table 3 $\left(0.6-2.3 \times 10^{8} \mathrm{yr}\right)$.

We also repeat that our black hole mass measurements produce dynamical masses of a central point source, which to our spatial resolution could be anything smaller than $\sim 25 \mathrm{pc}$. It is therefore plausible that some of the mass measured is in the accretion disk (or, perhaps, in a nearby reservoir feeding the accretion disk). If that is the case, any mass that has not accreted directly onto the black hole would not cause the black hole to turn on as an AGN. In such a scenario, the Eddington rate may not be a relevant limiting factor in this growth phase. 


\subsubsection{How Long Would It Take To Return to Scaling Relations?}

The gas-rich major mergers we have studied here appear to have black holes significantly larger than would be expected based on their host galaxy properties. We assume that these systems, post-merger, ought to fall on scaling relations. As our systems are currently undergoing enhanced star formation, it is likely that the host galaxies will indeed continue to grow over the course of the merger, moving back toward the local scaling relations in Figures 2-4. In Table 4, we show the relevant star formation rates from Howell et al. (2010) and calculate how long it would take at this star formation rate to bring the total stellar masses up to those predicted by the measured black hole masses.

We see that the typical timescale for these objects to reach predicted total stellar masses is $1-2 \mathrm{Gyr}$ at the present star formation rate. The dynamical models of Privon et al. (2013) find merger timescales for their sample of four systems to range from $140 \mathrm{Myr}-1.4 \mathrm{Gyr}$, depending on the initial conditions of the merger. Apart from IRASF10364-1042, our galaxies are not far from this range. Though star formation is a variable quantity, it is plausible for these galaxies to return to the $M_{\mathrm{BH}}-M_{* \text {,total }}$ relation by the time they have finished merging.

As noted above, it is possible that not all the mass we measured has been accreted by the black hole yet. If this is the case, it allows for the possibility that some of this mass might be ejected via AGN-driven winds and may indeed never be accreted; we discuss this scenario in Section 5.3. If this were to happen, the stellar mass growth need not be so high to return these systems to the $M_{\mathrm{BH}}-M_{* \text {,total }}$ relation.

\subsubsection{Is There Enough Gas Mass Left to Grow the Host Galaxy?}

Regardless of the rate of star formation discussed in Section 5.2.2, we might ask if there is enough molecular gas present in these galaxies to increase the $M_{* \text {,total }}$ sufficiently if all the gas were to be turned into stars eventually. We include the available mass in cold gas measured for each of these galaxies, when available, using $\mathrm{H}_{2}$ masses measured by Wilson et al. (2008). However, Wilson et al. (2008) point out that the CO-to- $\mathrm{H}_{2}$ conversion factor used is a primary source of uncertainty, and caution that their masses could be underestimated by up to a factor of 5 , and that AGN contamination in UGC 5101 could cause further underestimation. Taking these uncertainties into account, the gas masses could be sufficient in UGC 5101 and NGC 6240, but perhaps not in NGC 2623 or IRASF17207-0014.

We note that this estimate ignores atomic gas which may eventually cool and form molecular gas and then stars; this quantity could be substantial, and that these systems could also continue to accrete gas from the circumgalactic medium. However, a number of ULIRGs are also hosts to massive molecular outflows (Rupke \& Veilleux 2011; Sturm et al. 2011; Spoon et al. 2013; Veilleux et al. 2013), which could instead further deplete the gas.

Thus, the low gas mass estimates may support the possibility that not all excess mass measured from the central regions will eventually be accreted to the central black hole.

\subsection{Are These Masses Truly Measuring Black Holes?}

Our technique produces a dynamical measurement of the mass produced by a point source in the centers of these galaxies. Though the most straightforward interpretation of this is that the mass measured describes a black hole mass, it is possible that the mass distribution merely appears as a point source to our spatial resolutions. That is, anything significantly smaller than our pixel scales of $\sim 25 \mathrm{pc}$, and above the disk's radial density profile, would appear as a black hole to our measurements.

As discussed in Medling et al. (2011) and U et al. (2013), it is possible that some mass may be due to a nuclear star cluster. However, we rule out this interpretation for two reasons: (1) nuclear star clusters commonly have radii $\sim 100 \mathrm{pc}$ (Murray 2009), which would not appear as a point source to our dynamical modeling, and (2) the continuum luminosity present is not sufficient to account for a significant fraction of the central masses being due to stars. (Full spectral energy distribution fitting to the star clusters in and around the nuclear regions of these galaxies will be presented in a future paper.) We cannot, however, rule out the idea that gas present in the center could someday form a nuclear star cluster.

Galaxy merger simulations commonly assume Bondi-Hoyle accretion (Bondi \& Hoyle 1944; Bondi 1952; Springel et al. 2005b), a prescription in which effectively gas particles that enter within the sphere of influence of the black hole are automatically accreted so long as the Eddington rate is not exceeded. However, gas inflow likely has angular momentum which must be discarded before accretion can occur, which can delay the accretion event by, e.g., the viscous timescale (Power et al. 2011; Wurster \& Thacker 2013b). Though we do not have strong constraints on the properties of AGN accretion disks, estimates predict that viscosity could slow down accretion by more than a Hubble time (King 2008). Although the existence of AGN confirms that accretion can occur on shorter timescales, the delay may not be negligible. This allows for the possibility that gas can build up in the core of a galaxy around the black hole without having yet been accreted. This accretion disk (and, possibly, a reservoir feeding the accretion disk) of gas could contain a substantial amount of mass en route to the black hole. This mass would all appear as a point source to our dynamical models.

Given the scaling arguments in the sections above, we also note the possibility that this putative accreting gas may never make it to the black hole. Although the black hole dominates the gravitational pull on this gas, we find it plausible that when the black hole begins to accrete, its feedback may blow some of the rest of the gas away. Thus, the extra mass that we see here could be the reservoir for massive outflows seen in a variety of local AGN (e.g., Alatalo et al. 2011; Rupke \& Veilleux 2011; Sturm et al. 2011; Spoon et al. 2013; Veilleux et al. 2013). At mass outflow rates of tens or hundreds of solar masses per year, the excess mass could easily be ejected in the time remaining in the merger. It is not currently known where the mass carried in these outflows is drawn from, or in particular, how much of it might come from within $\sim 25 \mathrm{pc}$.

Simulations have also suggested that nuclear gas disks may leave behind remnant stellar disks on 1-10 parsec scales with masses 0.1-1 $M_{\mathrm{BH}}$ (Hopkins \& Quataert 2010b); if this were to occur, the final black hole masses in our sample will be decreased by $10-50 \%$. We note, however, that the black hole masses from which the scaling relations were calibrated did not have sufficient resolution to separate out remnant disks on 1-10 parsec scales either; thus we only introduce a bias if our sample is more likely to host such disks than the typical galaxy. We note that such remnant disks were discovered in galaxy merger 
simulations but that they did not form until after the final coalescence of the two nuclei. Thus it is not clear whether we would expect such disks (broken from the larger nuclear disk on hundred parsec scales) to be present in our sample.

Though the above possibilities may moderate the black hole masses measured by attributing some mass to other features, we still conclude that the black holes (combined with their accretion disks) in this sample of gas-rich mergers do not lie below black hole scaling relations, and in many cases are significantly more massive than their host galaxy properties would predict. This is directly opposite the predictions of quasar-mode feedback theories, in which black hole growth is delayed until the final stages of the galaxy merger when it cuts off star formation. As we note that (U)LIRGs are prototypically expected to evolve into quasars (Sanders \& Mirabel 1996), we find this as further evidence of some delay in feedback timescale during a merger. Such a delay (due, e.g., to a viscous accretion disk, as discussed above) could reconcile the latestage AGN feedback paradigm with the early mass growth presented here. Testing this scenario requires spatial resolutions higher by a factor of 10-20, which may be feasible in the upcoming era of thirty-meter-class telescopes.

\subsection{Comparison with Previous Findings}

These results differ from the conclusions drawn by Kormendy \& Ho (2013), who show five merging galaxies falling below the $M_{\mathrm{BH}}-\sigma_{*}$ and $M_{\mathrm{BH}}-L_{K \text {, bulge relations. They }}$ suggest that black hole growth might lag bulge formation in mergers, the opposite of the findings of this work. It is important to note the differing samples when considering these discrepant results. Our sample of merging galaxies contains major gas-rich mergers; the merging galaxies considered in Kormendy \& Ho (2013) are mainly minor mergers and/or gaspoor mergers. It is not unreasonable to imagine that such different types of mergers would have different effects on their central black holes. For example, a minor or gas-poor merger might use up all incoming new gas in star formation before it reaches the central black hole, causing a delay in black hole accretion until stellar feedback produces new gas to fuel it. A major gas-rich merger may have sufficient gas inflow to enable both star formation and black hole growth to begin relatively early.

A sample of black holes in submillimeter galaxies appear to fall below the locally defined $M_{\mathrm{BH}}-M_{* \text {, bulge relation by } 1-2}$ orders of magnitude (Borys et al. 2005; Alexander et al. 2008). These galaxies are thought to be the high-redshift $(z \sim 2)$ counterparts of local ULIRGs and therefore might be reasonable to expect that they would behave in the same way as the sample presented here. However, the discrepancy may in fact be due to their usage of broad line velocity dispersions to infer $M_{\mathrm{BH}}$; indeed, when applying the same method in one local ULIRG, Alexander et al. (2008) find rough agreement with their submillimeter galaxies. It is also possible that differences between the samples such as Eddington ratio, redshift, and merger stage might affect the black hole growth timescales.

\section{CONCLUSIONS}

We have presented high spatial resolution near-infrared integral field spectroscopy of nuclear disks in nearby merging (U)LIRGs. These gas-rich mergers funnel gas to the galaxy centers where the gas forms disks; stars then form in situ, creating a stellar disk as well. These disks, which were described in detail in Medling et al. (2014), provide an alternative method to measuring black hole masses. Threeintegral orbital superposition models rely on an assumption of virialization and emission line black hole mass diagnostics require broad lines; neither of these methods is feasible in such systems. However, with adaptive optics, we have produced two-dimensional kinematic maps at spatial resolutions close to or better than radius of the sphere of influence of the black hole. Because the dynamical timescale this close to a black hole is short, the material quickly becomes virialized, and kinematic modeling is possible.

We implemented two different techniques using these kinematic maps, originally presented in Medling et al. (2011) and $U$ et al. (2013), to measure nine new black hole masses, producing a sample of black hole masses in nine merging systems. By obtaining both gas and stellar kinematics for many of these systems, we are able to provide multiple independent measurements for a large fraction of our sample. Gaseous disks are modeled as thin Keplerian disks. Stellar disks are more likely to have pressure support, but measuring this is not straightforward, so we place bracketing measurements using two assumptions. To provide a lower limit to the black hole mass, we assume the stars are also in a thin Keplerian disk; any pressure support would increase the black hole mass. We also use JAM models to take into account the velocity dispersion; since this velocity dispersion may be inflated by intervening unvirialized material along the line of sight, this mass measurement is an upper limit.

As the black hole masses measured here are in fact masses enclosed in $\sim 25 \mathrm{pc}$, some of the mass attributed to black holes here may be gas that has not yet accreted onto the black hole. Although this mass is gravitationally bound to the black holes, we note that it is possible that future AGN-driven outflows may carry a fraction of it away, thus diminishing the final black hole mass. Though such outflows have now been seen in a number of (U)LIRGs, further studies will reveal how frequently they occur, how much mass they carry, and how much of the loaded mass comes from within $\sim 25 \mathrm{pc}$.

We have placed these enclosed masses on three local black hole scaling relations, $M_{\mathrm{BH}}-\sigma_{*}, M_{\mathrm{BH}}-L_{H \text {,bulge }}$, and $M_{\mathrm{BH}}-M_{*}$, using host galaxy parameters from the literature. We find that though individual galaxies often fall within the scatter, as a population they are significantly offset toward higher black hole masses than isolated systems. Several systems have central masses that sit considerably above scaling relations.

Under the assumption that these galaxies follow black hole scaling relations before and after their mergers, this suggests that black holes grow more quickly than their host galaxies during a major gas-rich merger. This does not line up with theoretical reasoning that star formation has easier access to inflowing gas (e.g., Cen 2012; Hopkins 2012) or that a final burst of black hole growth is likely to shut off star formation ("quasar-mode feedback"; Hopkins et al. 2005; Springel et al. 2005a), unless that feedback is delayed due to viscosity in the accretion disk (Power et al. 2011; Wurster \& Thacker 2013b) or similar. We conclude that black hole fueling begins early in a gas-rich merger, and can initially outpace any simultaneous starbursting or bulge formation.

These results have focused on a sample of gas-rich mergers in late stages of merging. To trace black hole growth through the entire merger sequence, a larger sample of black hole 
masses should be obtained for systems at a range of merger stages. Such an observational sample could provide the basis for distinguishing between prescriptions for black hole accretion and feedback physics in galaxy merger simulations (e.g., Wurster \& Thacker 2013a).

A.M.M. acknowledges useful conversations with George Privon, Sebastian Haan, Tiantian Yuan, Stephanie Juneau, Kevin Schawinski, David Rosario, and Simona Gallerani. We enthusiastically thank the staff of the W. M. Keck Observatory and its AO team, for their dedication and hard work. Data presented herein were obtained at the W. M. Keck Observatory, which is operated as a scientific partnership among the California Institute of Technology, the University of California, and the National Aeronautics and Space Administration. The Observatory and the Keck II Laser Guide Star AO system were both made possible by the generous financial support of the W. M. Keck Foundation. The authors wish to extend special thanks to those of Hawai'ian ancestry on whose sacred mountain we are privileged to be guests. Without their generous hospitality, the observations presented herein would not have been possible. This work was supported in part by the National Science Foundation Science and Technology Center for Adaptive Optics, managed by the University of California at Santa Cruz under cooperative agreement AST 98-76783. This material is based in part upon work supported by the National Science Foundation under award number AST-0908796.

Facility: Keck:I (Laser Guide Star Adaptive Optics OSIRIS), Keck:II

\section{APPENDIX A \\ DYNAMICAL MASS MODELS}

\section{A.1 Fits and Residuals}

In this section, we show the best-fit dynamical mass models for each galaxy for each modeling technique in Figures A1
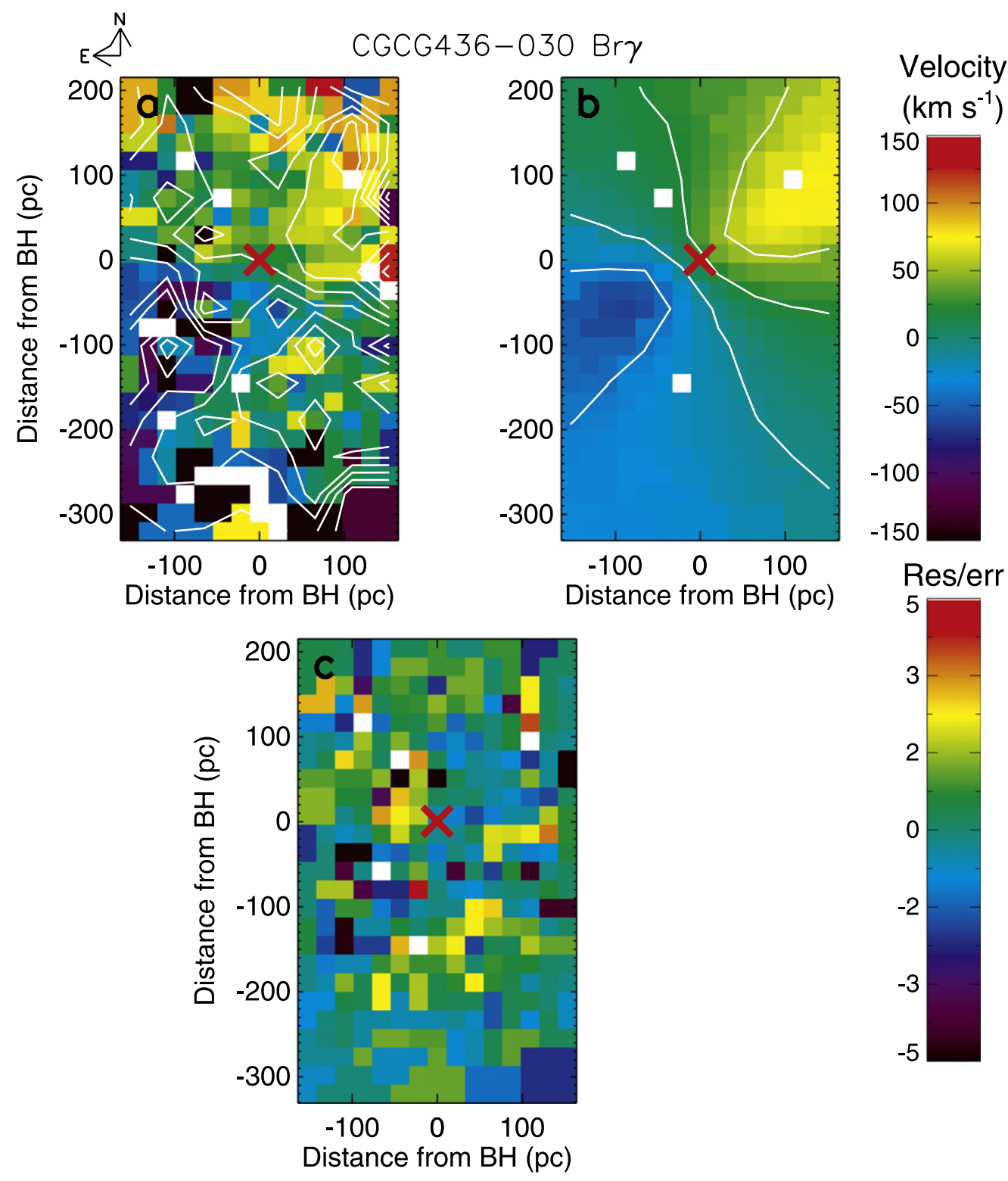

Figure A1. (a) Map of Br $\gamma$ velocities of the inner region of CGCG436-030. (b) Map of model velocities of best fit black hole model. (c) Residual map of best-fit model divided by errors in velocity. In panels (a) and (b), velocity contours are marked in white. In each panel, a red X marks the position of the black hole. 


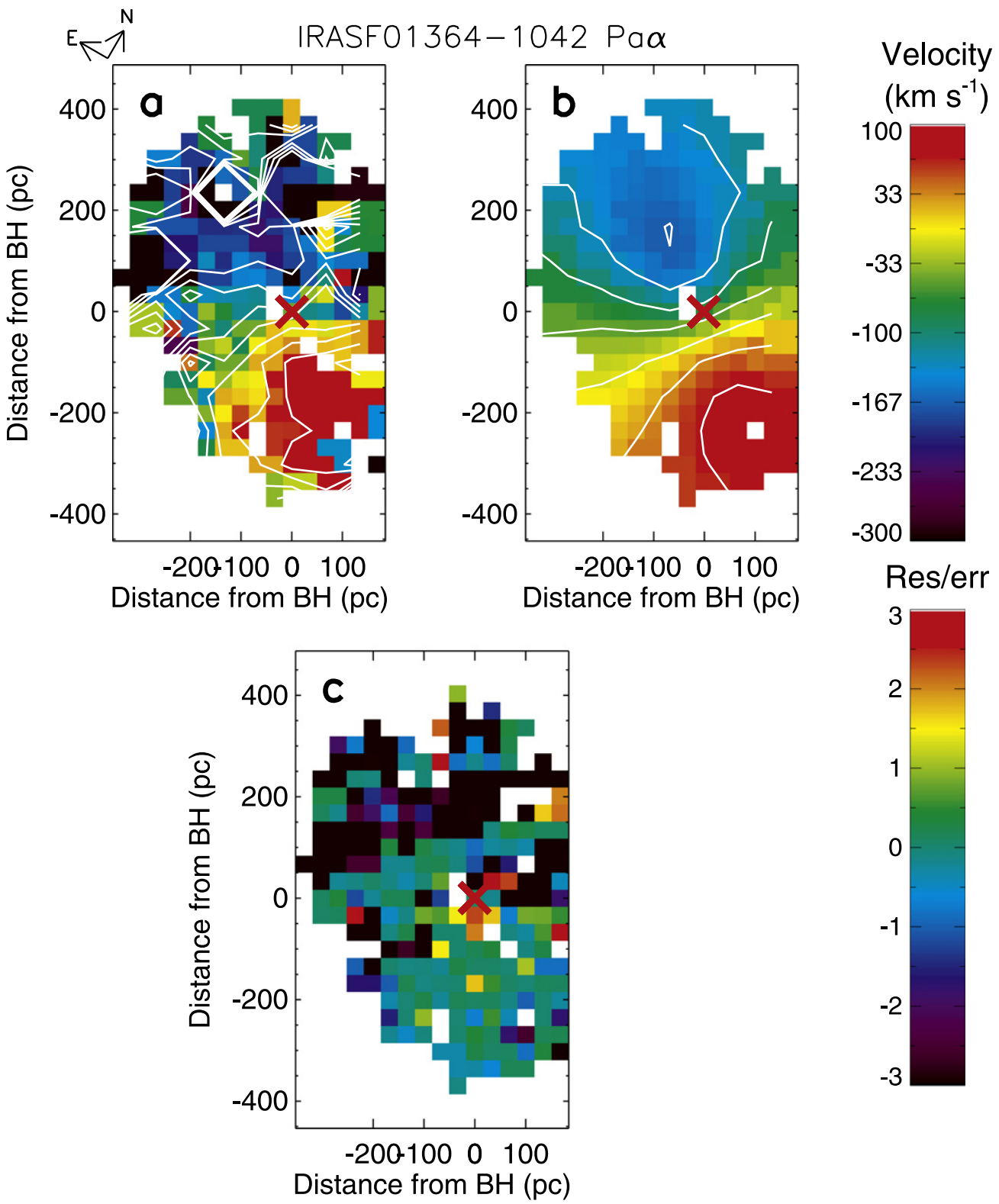

Figure A2. (a) Map of Pa $\alpha$ velocities of the inner region of IRASF01364-1042. (b) Map of model velocities of best fit black hole model. (c) Residual map of best-fit model divided by errors in velocity. In panels (a) and (b), velocity contours are marked in white. In each panel, a red X marks the position of the black hole.

-A18. As discussed in Section 4, each black hole was dynamically fit using a subset of the following methods: thin disk fitting to $\mathrm{Br} \gamma, \mathrm{Pa} \alpha$, or [Fe II] velocity map, thin disk fitting to $\mathrm{H}_{2}$ velocity map, thin disk fitting to stellar velocity map, JAM modeling to stellar velocity and velocity dispersion maps. Galaxies with multiple tracers show reasonable agreement in measured masses, which can be seen in Table 2 and Figures 2-4. Residuals from each fit, divided by the measurement error, are included in these figures and demonstrate the goodness-of-fit of each model. The fitted disk parameters are also given in Table A1.

We note that many galaxies have complex kinematic structures that deviate from pure rotation in one or more of the tracers. These can be due to tidal streams or spiral arms along the LOS, or to inflow or outflow when seen in gas tracers. Where possible, we have masked these out to avoid biasing the disk models. Masked spaxels appear white in the figures. A full analysis of the deviations from pure rotation and subsequent implications for inflow or outflow will be presented in a future paper.

The positions of the black holes, plotted at $(0,0)$ in each of the following figures, were determined primarily from the kinematic centers rather than the photometric centers. However, in the case of IRASF17207-0014, the kinematics are sufficiently complex that the two black holes were fixed to the photometric center of each disk measured with GALFIT in Medling et al. (2014).

\section{A.2 Black Hole Spheres of Influence}

One key feature of our thin disk black hole mass models is the inclusion of a radially varying mass component to incorporate the mass profile of the nuclear disk. (Our JAM models also incorporate galactic mass, but by fitting a mass-to- 


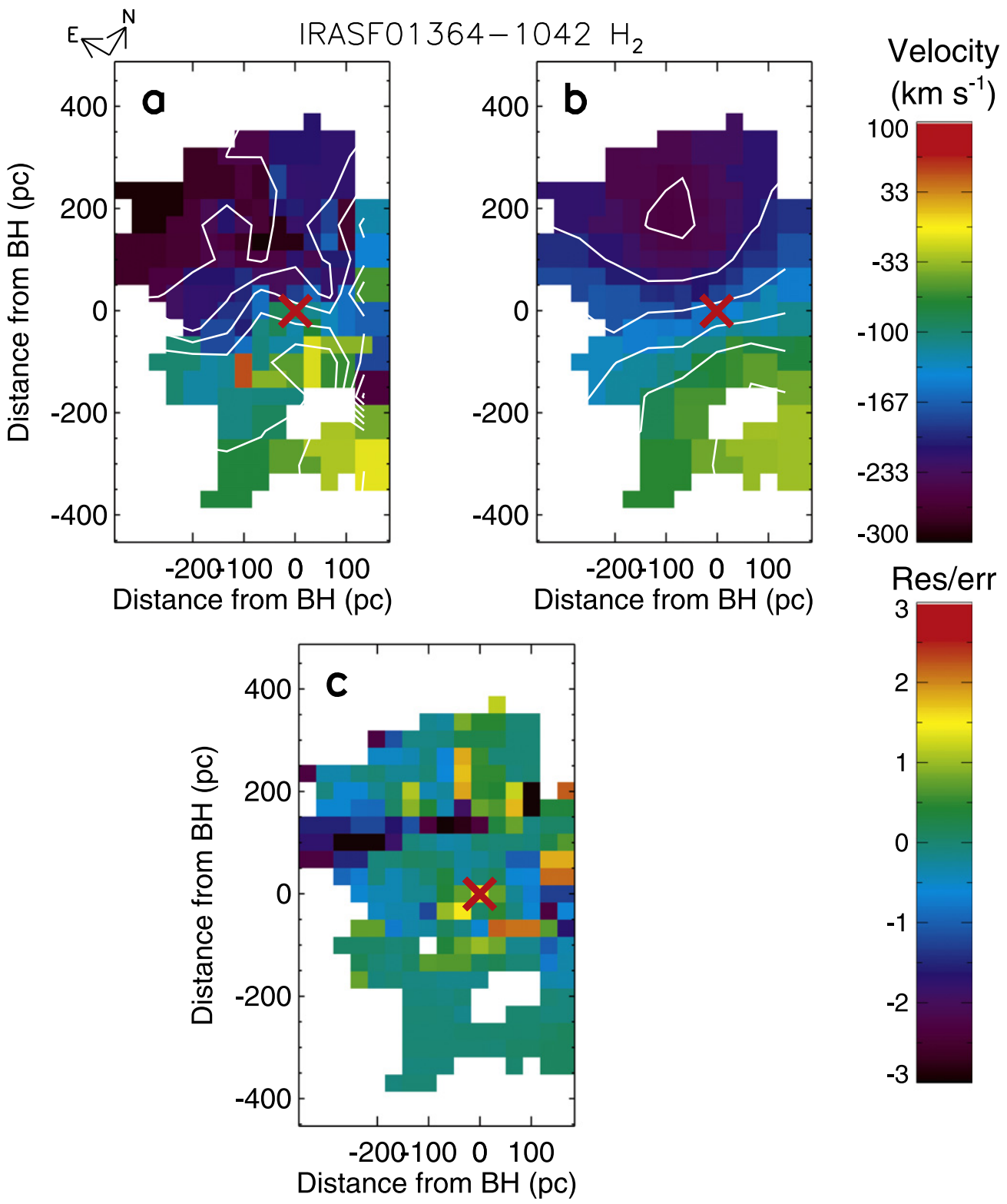

Figure A3. (a) Map of $\mathrm{H}_{2}$ velocities of the inner region of IRASF01364-1042. (b) Map of model velocities of best fit black hole model. (c) Residual map of best-fit model divided by errors in velocity. In panels (a) and (b), velocity contours are marked in white. In each panel, a red X marks the position of the black hole.

light ratio.) We note that our fits use only a single power-law fit to the mass profile, so any pile-up of matter below our resolution limit will be considered part of the point mass. We include the mass profiles measured here for completeness, and for the purposes of calculating the implied radius of the sphere of influence of each black hole. Recall that the mass profile is fit with the form $M(r)=\rho_{0} r^{-\gamma}$. In Table A1 we report the normalization and power law fit for each of these parameters. For clarity, instead of reporting $\rho_{0}$, we report the mass in the disk enclosed at 100 parsecs.

A black hole's sphere of influence is defined as the region within which the black hole contributes $50 \%$ of the mass. We can therefore use our thin disk mass profiles to calculate the radius of the implied sphere of influence. This is interesting both for the purposes of understanding black hole dynamics and for confirming that we indeed have sufficient resolution to measure the black hole masses appropriately.

As the fitted mass profiles of the disk and black hole masses are not independent, there is an issue of covariance to consider. That is, when a model fits a higher black hole mass, it will likely put less mass in the disk, and vice versa. When calculating errors on each of these parameters, we marginalize over the others: our black hole mass errors are calculated from the width of the black hole mass distribution in our Monte Carlo simulation, taking into account all possible fitted disk profiles. Similarly, we report our errors on disk profile parameters using the widths of the distributions taking into account all fitted black hole masses. This is an appropriate way to handle covariance when drawing conclusions about the black hole masses, as is done in this paper. To account for the covariance issue in the measurement of $r_{\text {infl }}$, we calculate $r_{\text {infl }}$ 

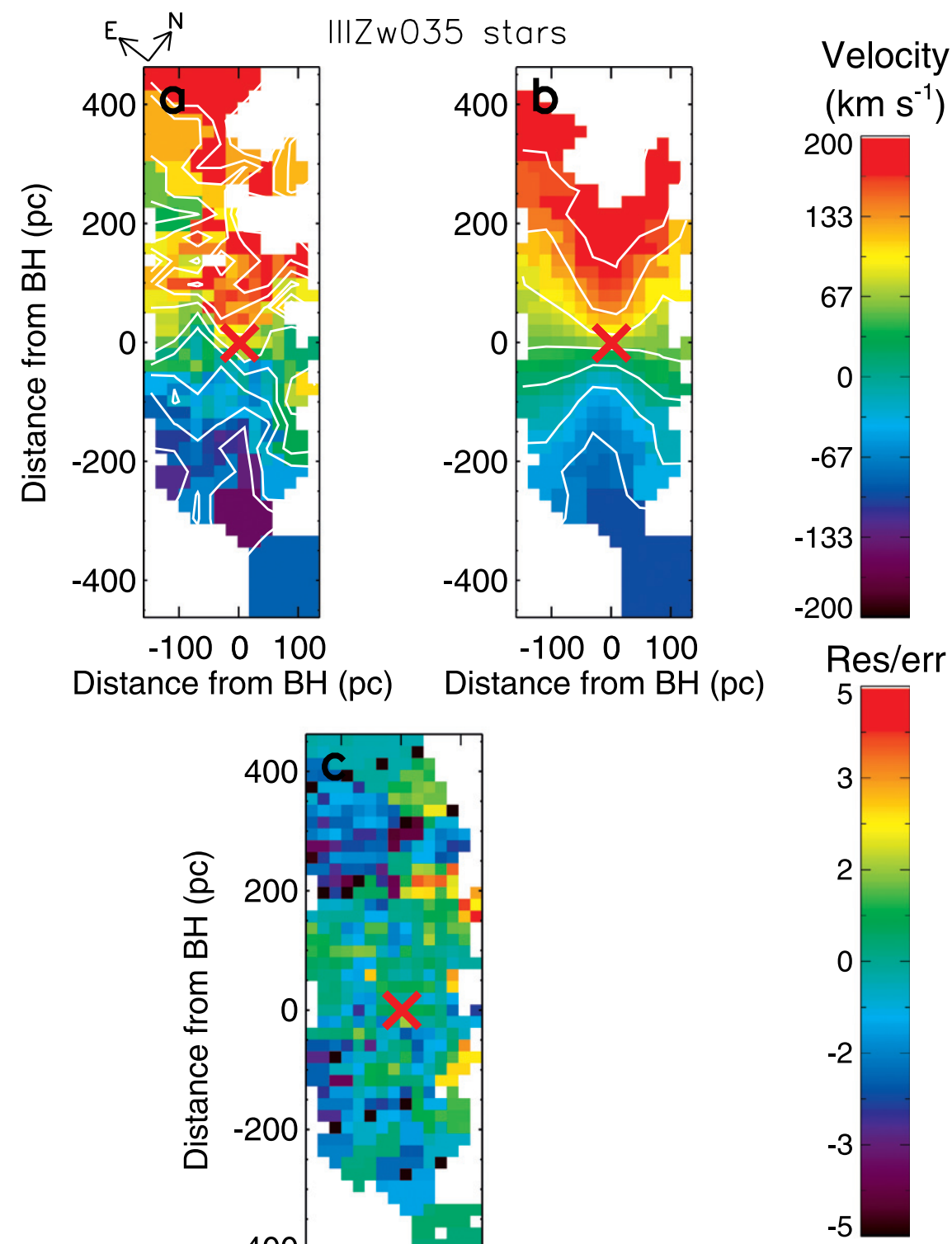

$-100 \quad 0 \quad 100$

Distance from $\mathrm{BH}(\mathrm{pc})$

Figure A4. (a) Map of stellar velocities of the inner region of IIIZw035. (b) Map of model stellar velocities of best fit black hole model. (c) Residual map of best-fit model divided by errors in velocity. In panels (a) and (b), velocity contours are marked in white. In each panel, a red X marks the position of the black hole.
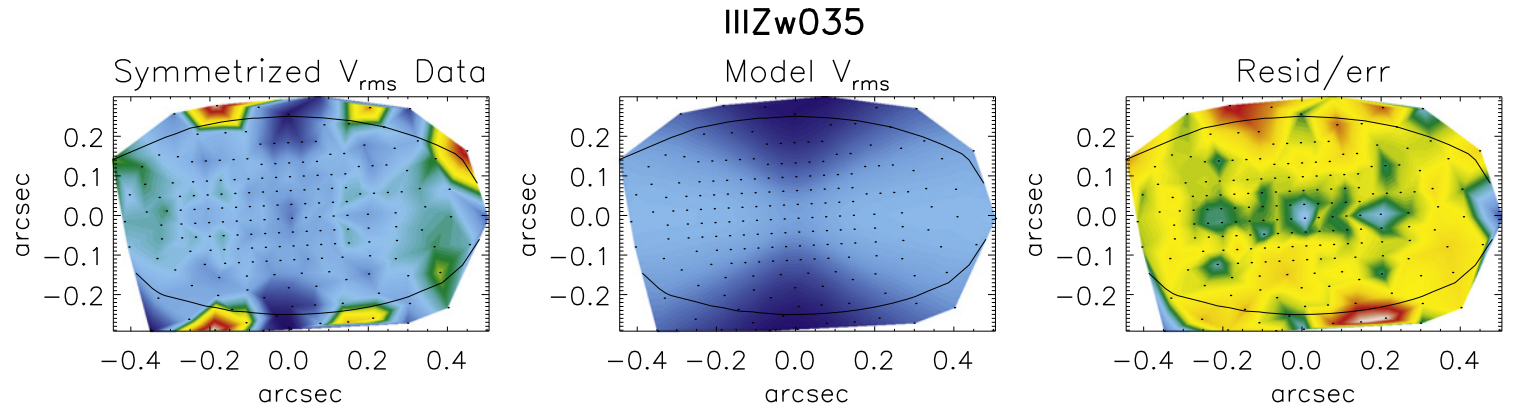

$\mathrm{km} \mathrm{s} \mathrm{s}^{-1}$
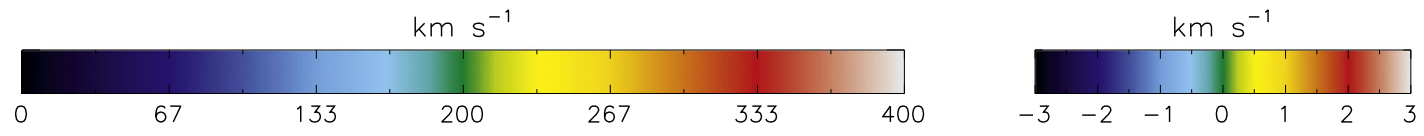

Figure A5. Left: map of the symmetrized $v_{\mathrm{rms}}=\left(v^{2}+\sigma^{2}\right)^{0.5}$ of IIIZw035. Center: model $v_{\mathrm{rms}}$ of galaxy with black hole. Right: residual map of model-data divided by errors in $v_{\text {rms. }}$. All three panels are centered on the black hole and have been rotated so the major axis is horizontal. 

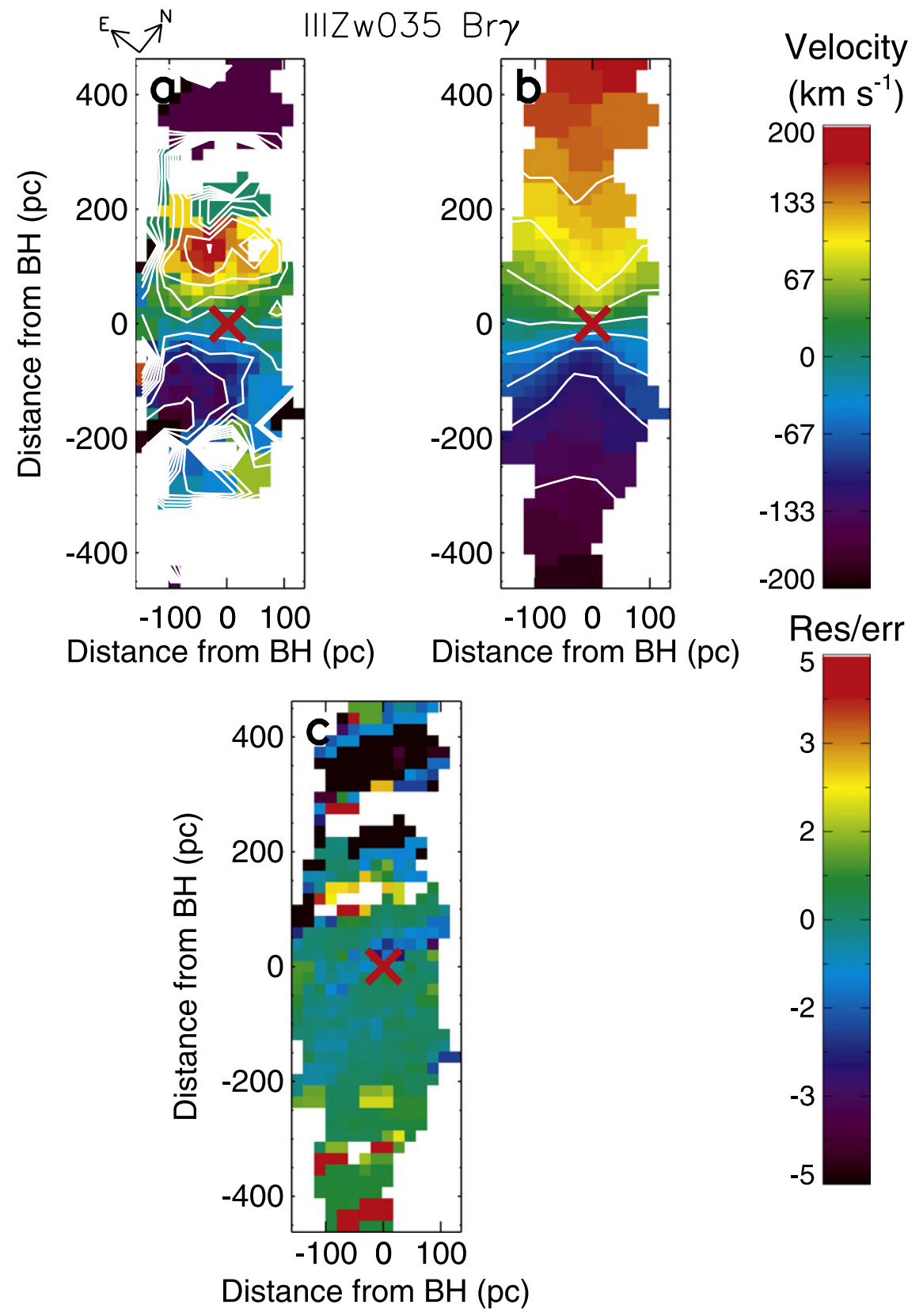

Figure A6. (a) Map of Br $\gamma$ velocities of the inner region of IIIZw035. (b) Map of model velocities of best fit black hole model. (c) Residual map of best-fit model divided by errors in velocity. In panels (a) and (b), velocity contours are marked in white. In each panel, a red X marks the position of the black hole.

for every fit in the Monte Carlo simulation and draw our conclusions based on that.

In one case (IRASF01364-1042), the black hole mass is comparable to or larger than the entire nuclear disk. The calculated radius of influence is thus artificially large, because presumably the mass profile of the galaxy changes beyond our FOV. The $\mathrm{H}_{2}$ fit to IIIZw035 is also artificially large; we attribute this to streaming motion in the $\mathrm{H}_{2}$ emission compromising the larger scale fit. This and other evidence of outflows will be presented in an upcoming paper. The affected radii are reported in parentheses in Table A1.

We see that, for our sample, we clearly resolve the spheres of influence of these black holes. We note that this method of calculating the size of the sphere of influence is uncommon, as a full mass profile is required to determine where $M(r)=M_{\mathrm{BH}}$. Our capacity to resolve these scales exceeded our expectations because the black holes are more massive than predicted. 


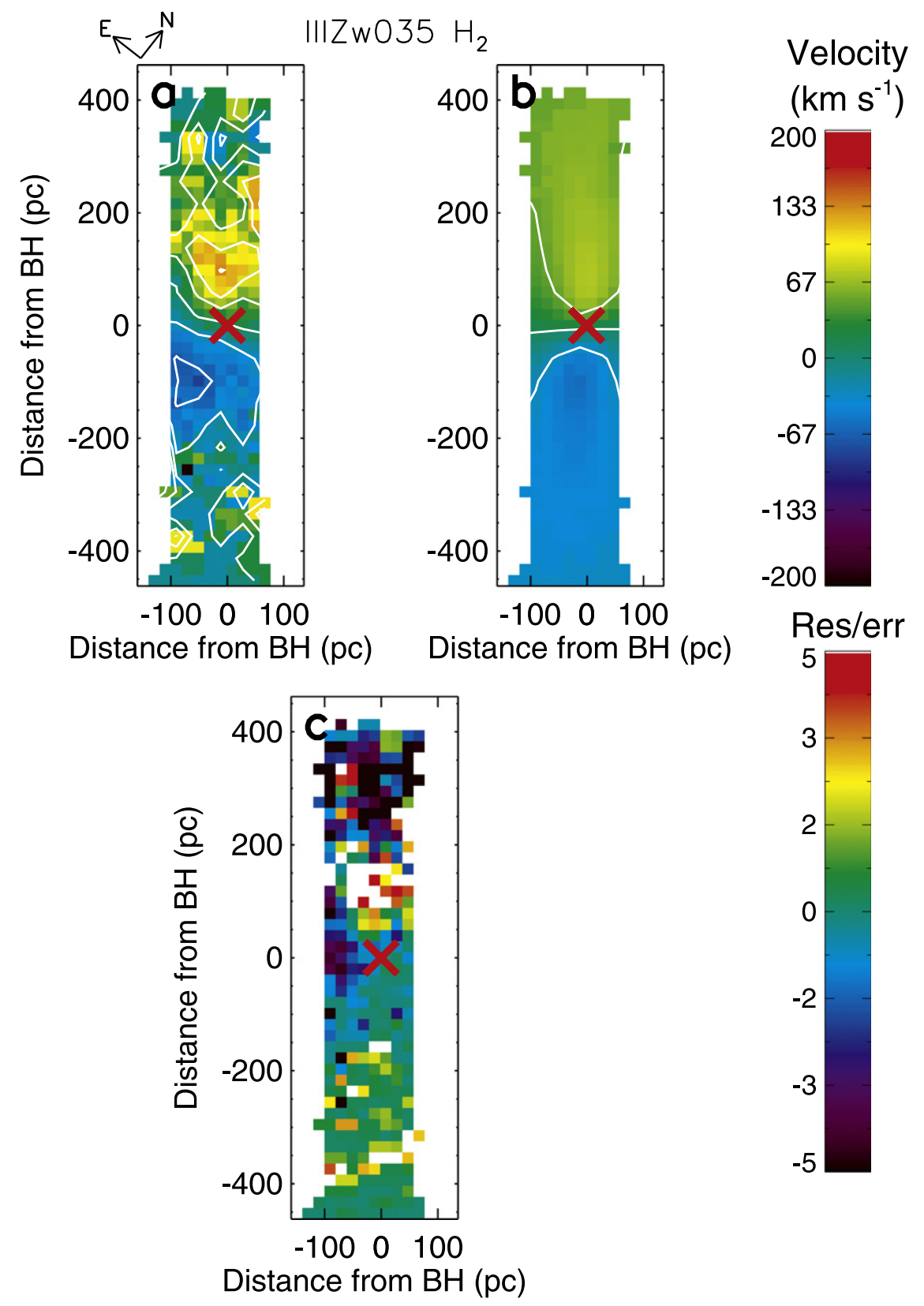

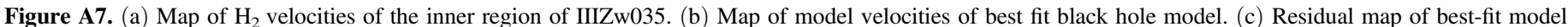
divided by errors in velocity. In panels (a) and (b), velocity contours are marked in white. In each panel, a red X marks the position of the black hole. 

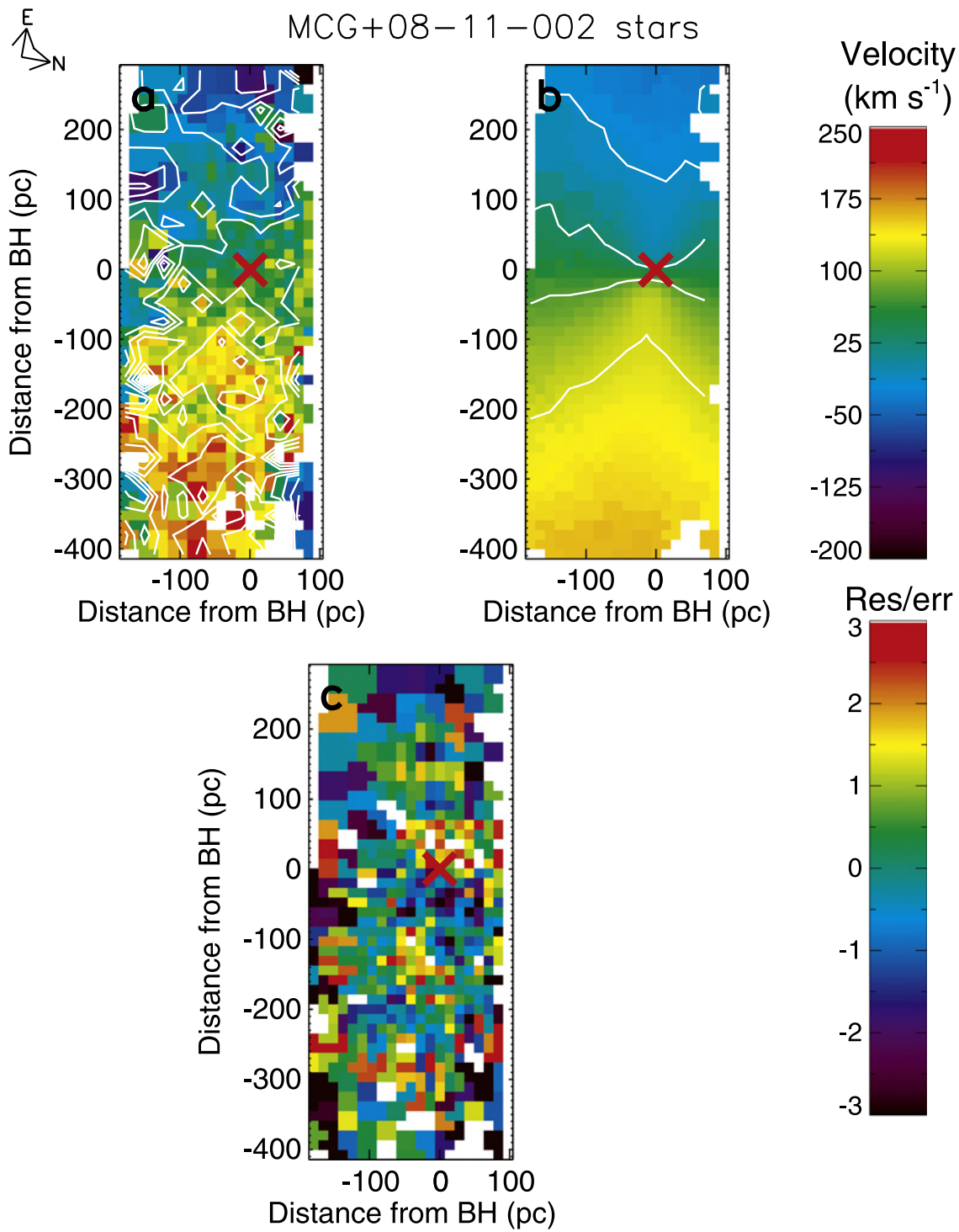

Figure A8. (a) Map of stellar velocities of the inner region of MCG+08-11-002. (b) Map of model velocities of best fit black hole model. (c) Residual map of best-fit model divided by errors in velocity. In panels (a) and (b), velocity contours are marked in white. In each panel, a red X marks the position of the black hole.

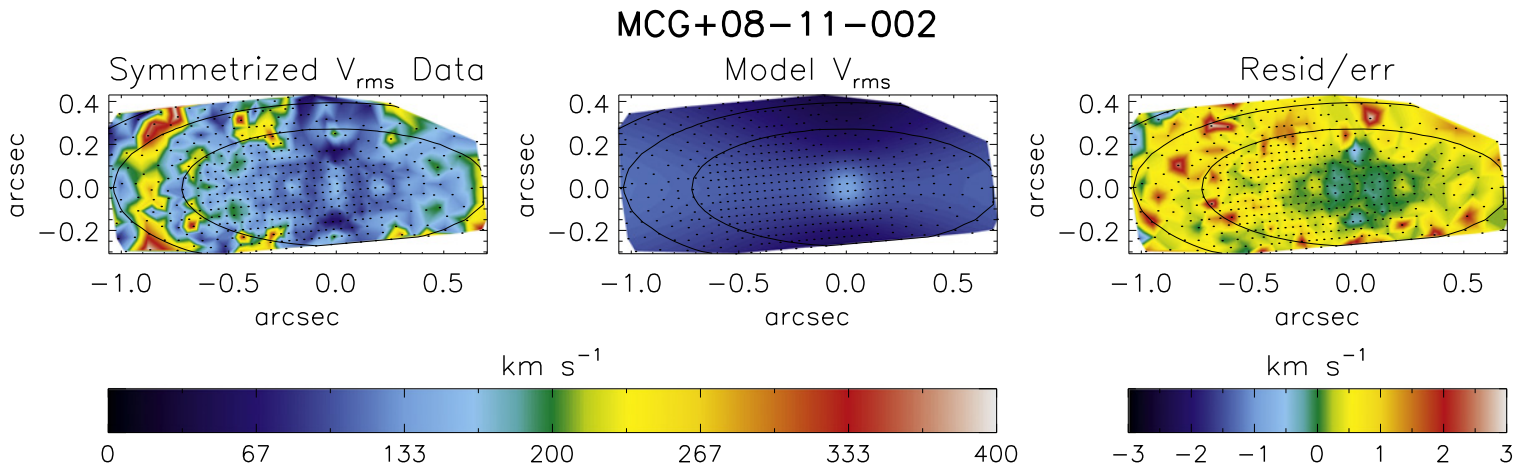

Figure A9. Left: map of the symmetrized $v_{\mathrm{rms}}=\left(v^{2}+\sigma^{2}\right)^{0.5}$ of MCG+08-11-002. Center: model $v_{\text {rms }}$ of galaxy with black hole. Right: residual map of model—data divided by errors in $v_{\text {rms. }}$. All three panels are centered on the black hole and have been rotated so the major axis is horizontal. 

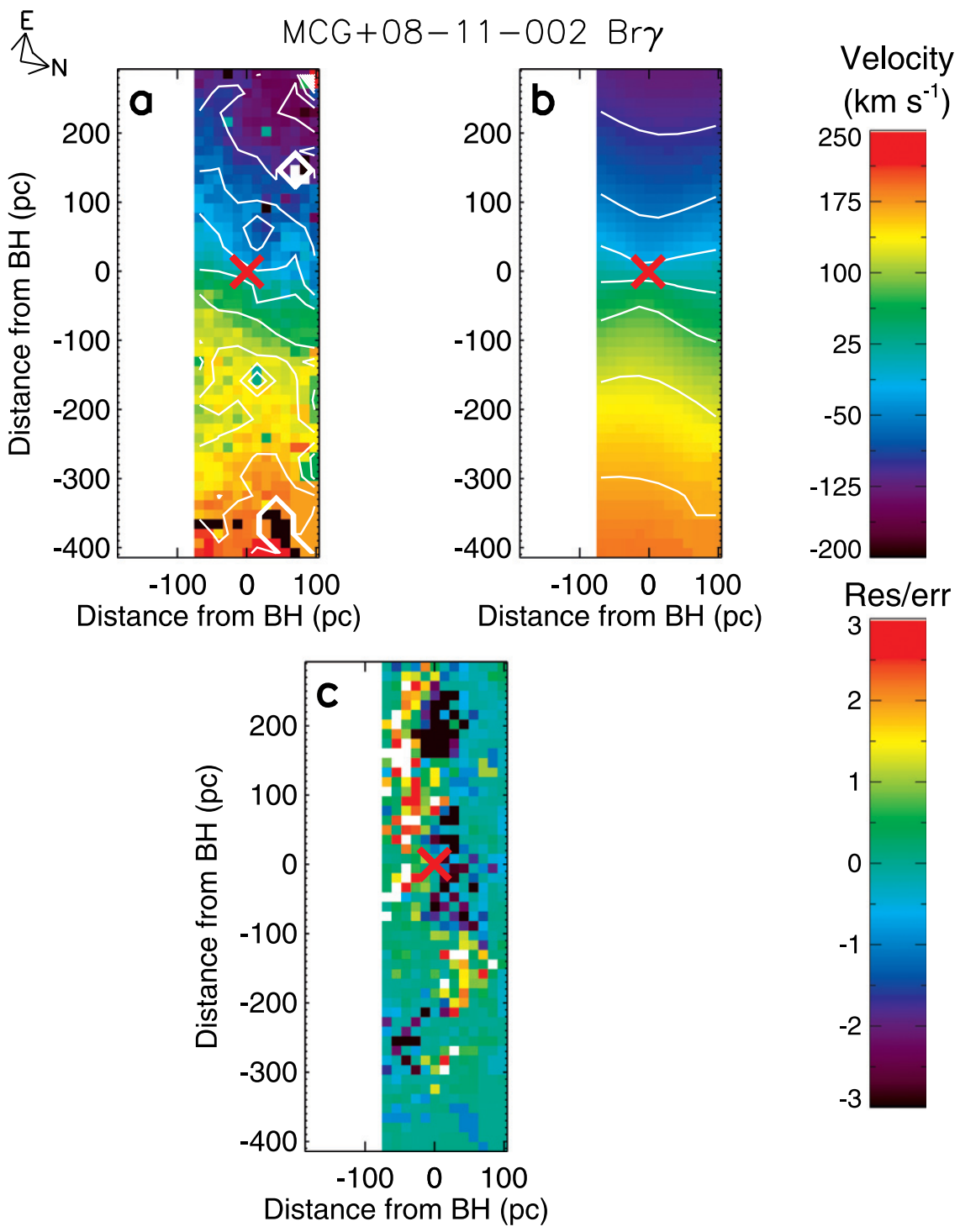

Figure A10. (a) Map of Br $\gamma$ velocities of the inner region of MCG+08-11-002. (b) Map of model velocities of best fit black hole model. (c) Residual map of best-fit model divided by errors in velocity. In panels (a) and (b), velocity contours are marked in white. In each panel, a red X marks the position of the black hole. 

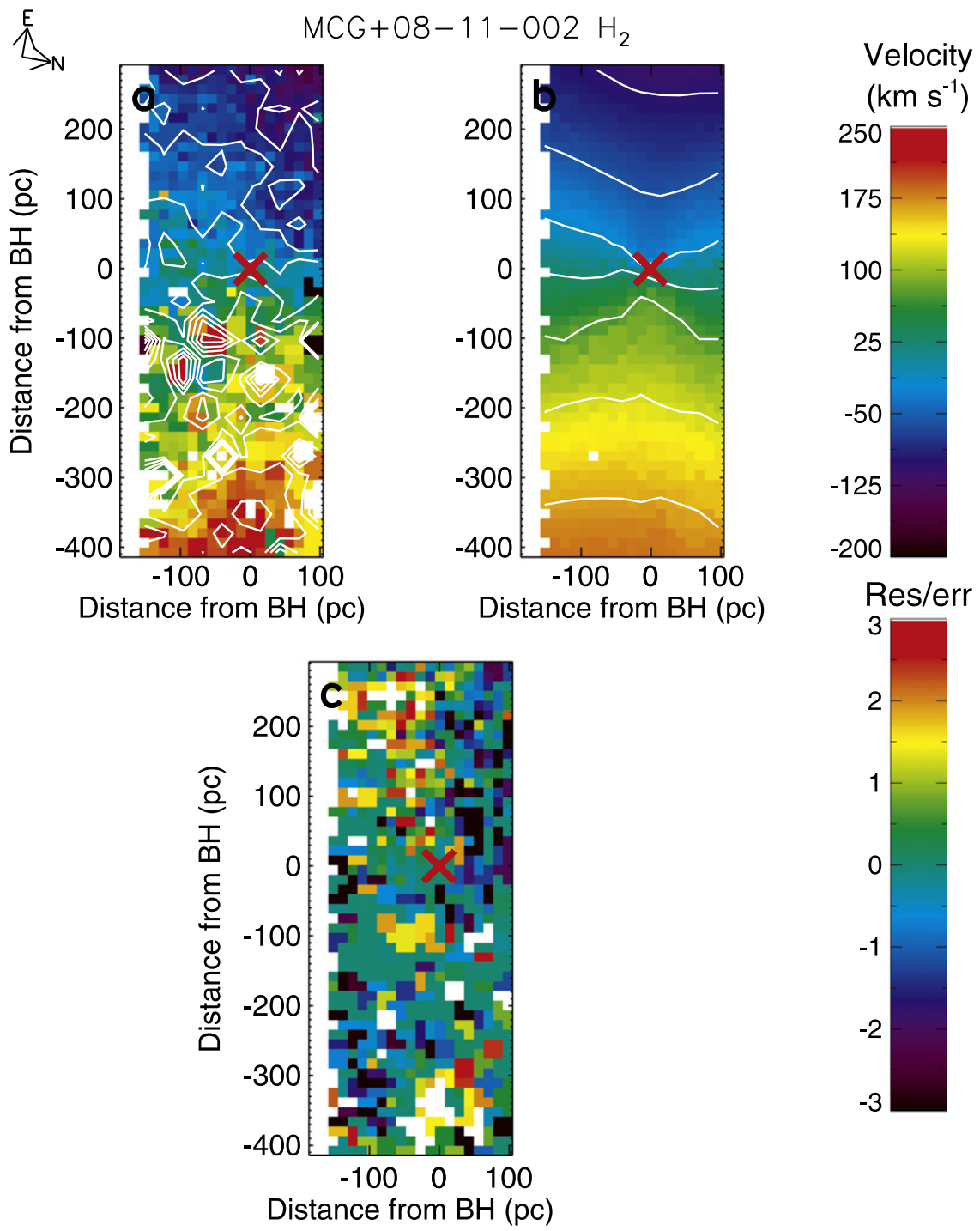

Figure A11. (a) Map of $\mathrm{H}_{2}$ velocities of the inner region of MCG+08-11-002. (b) Map of model velocities of best fit black hole model. (c) Residual map of best-fit model divided by errors in velocity. In panels (a) and (b), velocity contours are marked in white. In each panel, a red X marks the position of the black hole. 


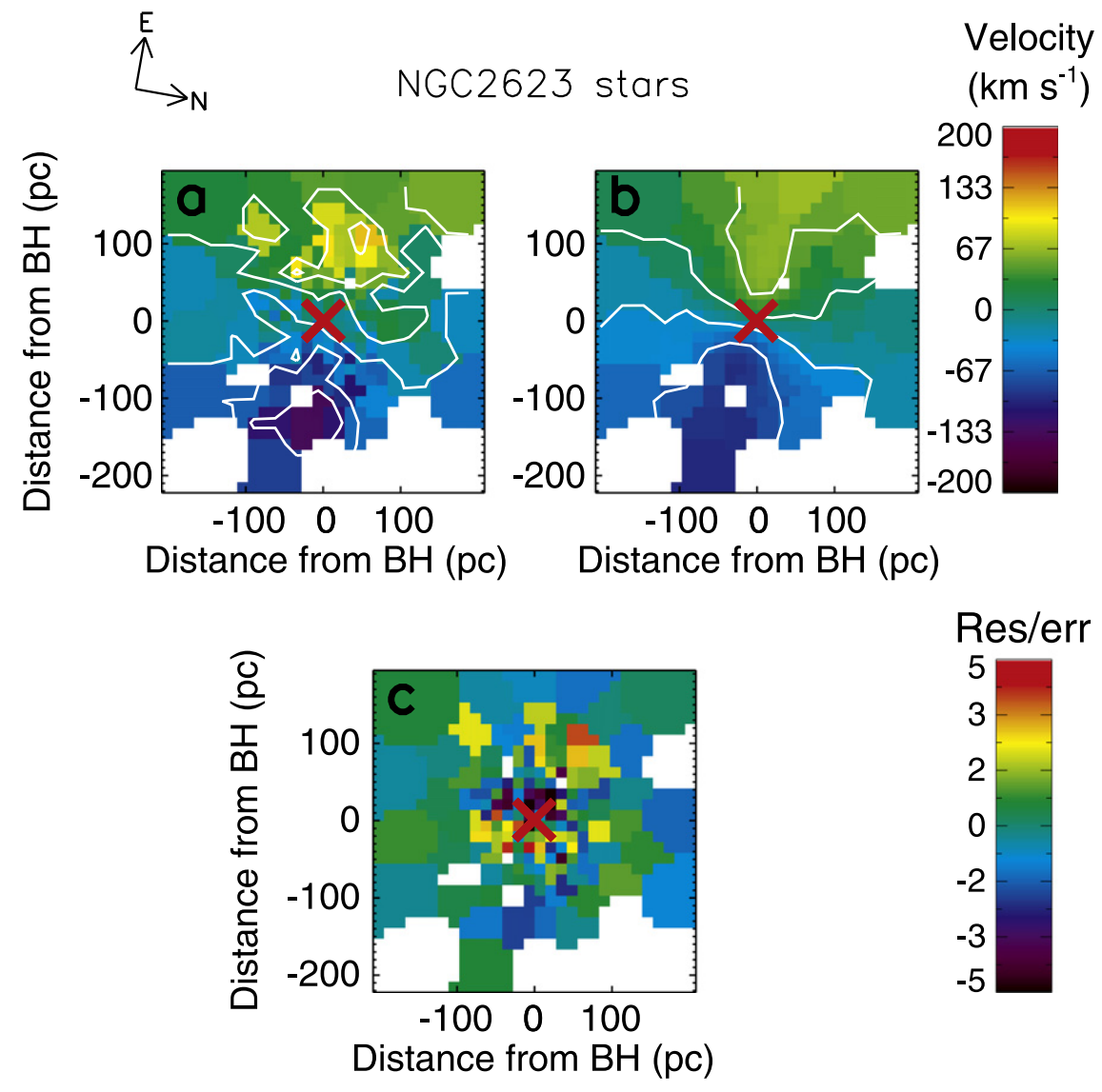

Figure A12. (a) Map of stellar velocities of the inner region of NGC 2623. (b) Map of model stellar velocities of best fit black hole model. (c) Residual map of best-fit model divided by errors in velocity. In panels (a) and (b), velocity contours are marked in white. In each panel, a red X marks the position of the black hole.

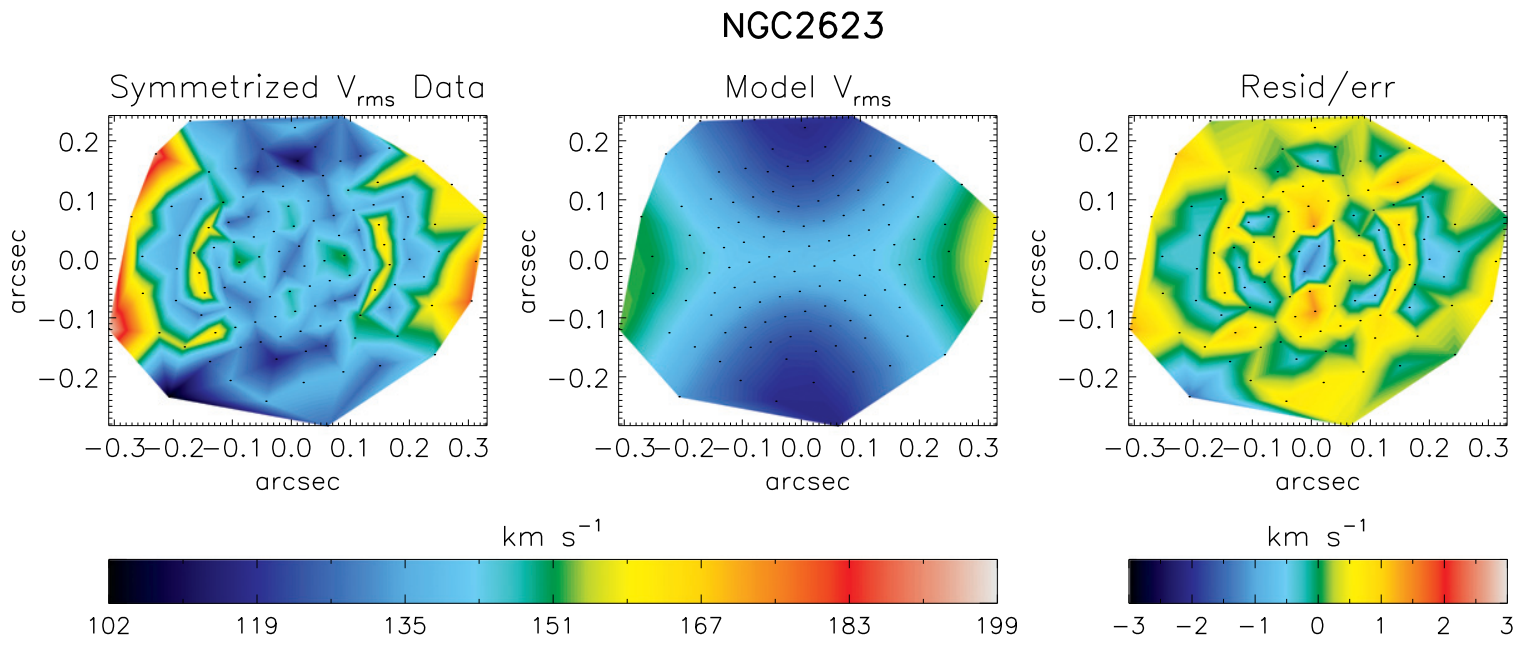

Figure A13. Left: map of the symmetrized $v_{\mathrm{rms}}=\left(v^{2}+\sigma^{2}\right)^{0.5}$ of NGC 2623. Center: model $v_{\text {rms }}$ of galaxy with black hole. Right: residual map of model- data divided by errors in $v_{\text {rms. }}$. All three panels are centered on the black hole and have been rotated so the major axis is horizontal. 

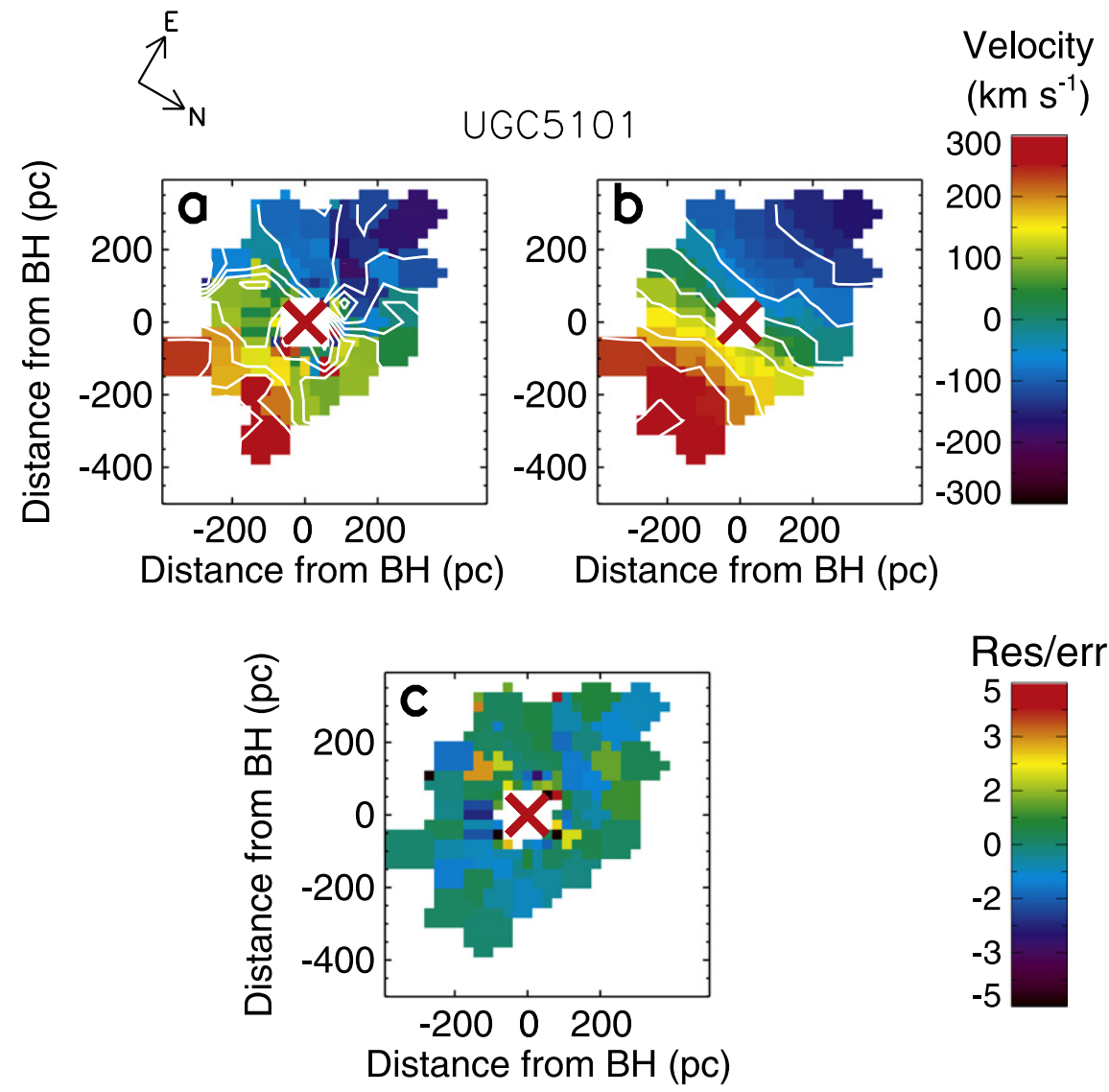

Figure A14. (a) Map of stellar velocities of the inner region of UGC 5101. (b) Map of model stellar velocities of best fit black hole model. (c) Residual map of best-fit model divided by errors in velocity. In panels (a) and (b), velocity contours are marked in white. In each panel, a red X marks the position of the black hole.

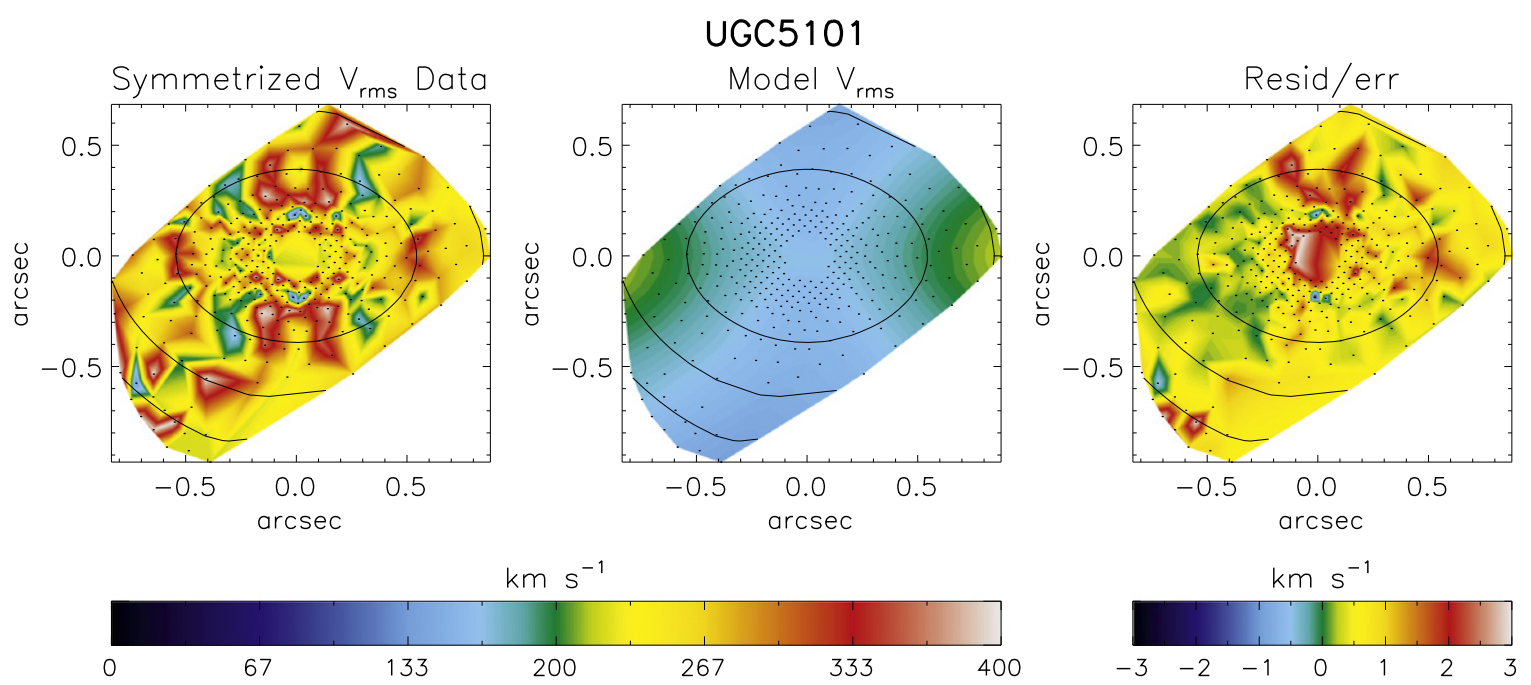

Figure A15. Left: map of the symmetrized $v_{\mathrm{rms}}=\left(v^{2}+\sigma^{2}\right)^{0.5}$ of UGC 5101. Center: model $v_{\mathrm{rms}}$ of galaxy with black hole. Right: residual map of model—data divided by errors in $v_{\text {rms. }}$. All three panels are centered on the black hole and have been rotated so the major axis is horizontal. 


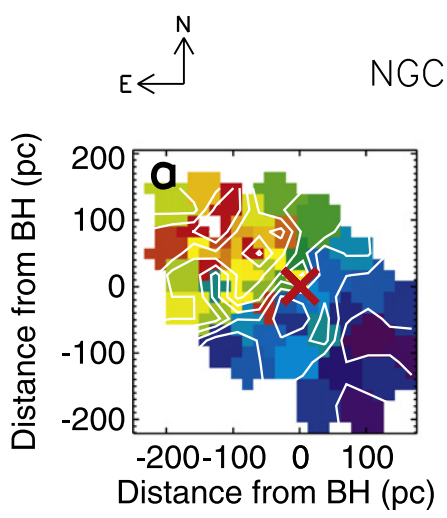

\section{NGC6240N}

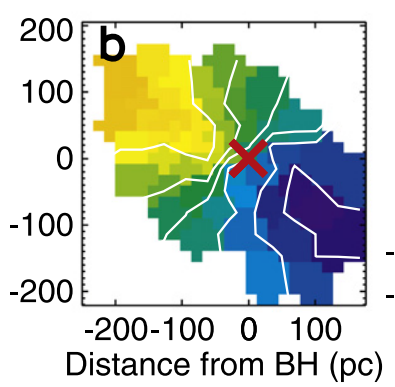

\section{Velocity \\ $\left(\mathrm{km} \mathrm{s}^{-1}\right)$}

200

133

67

0

$-67$

$-133$

$-200$
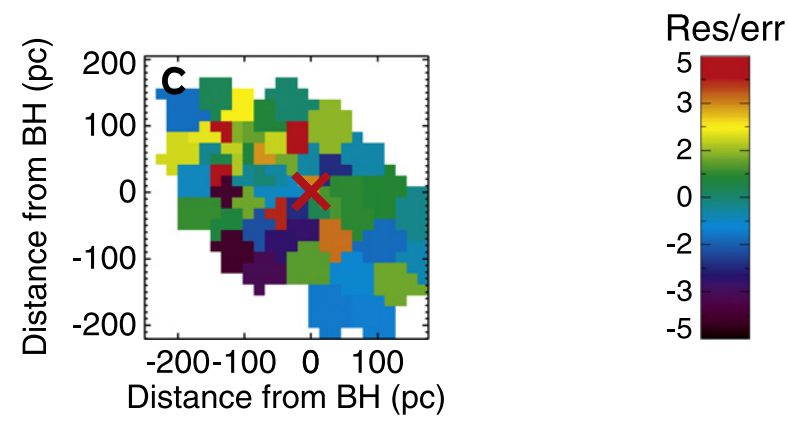

Figure A16. (a) Map of stellar velocities of the inner region of NGC 6240 N. (b) Map of model stellar velocities of best fit black hole model. (c) Residual map of best-fit model divided by velocity errors. In panels (a) and (b), velocity contours are marked in white. In each panel, a red X marks the position of the black hole.

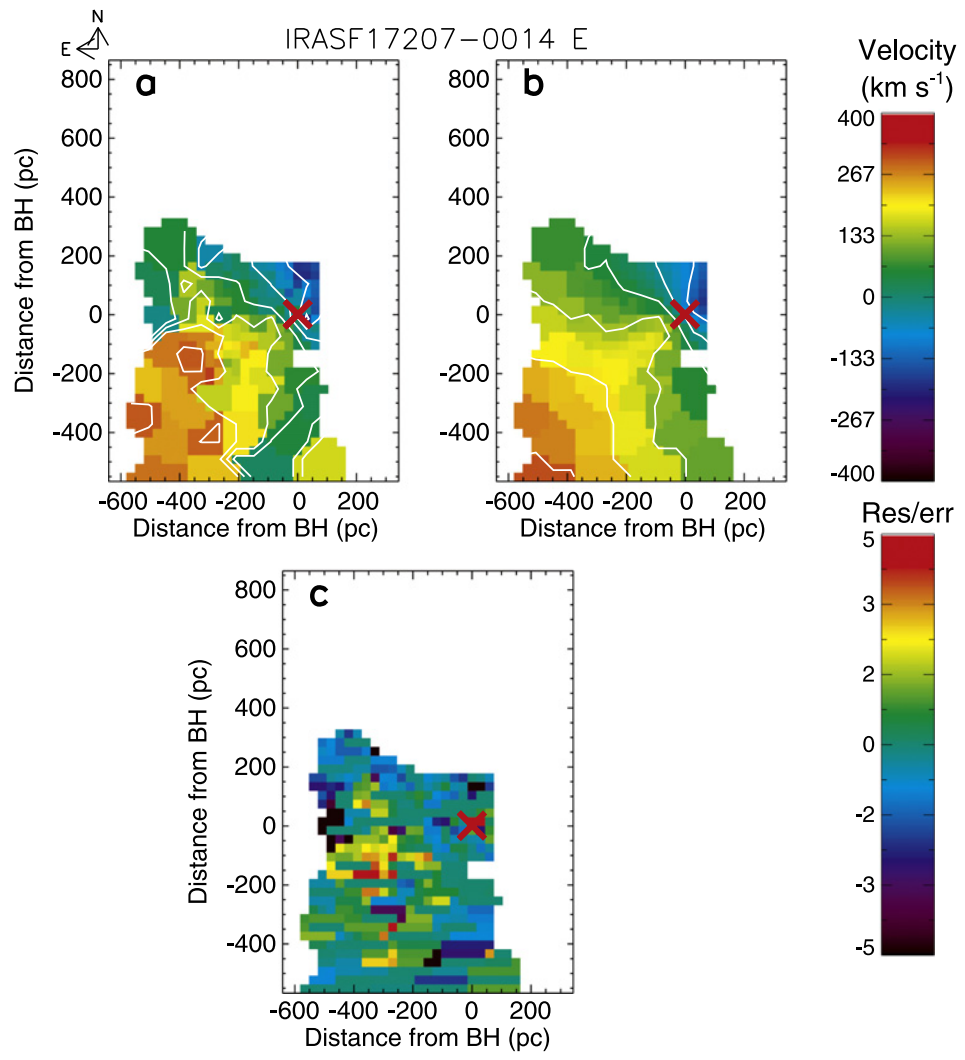

Figure A17. (a) Map of [Fe II] velocities of the inner region of IRAS F17207-0014. (b) Map of model velocities of best fit black hole model. (c) Residual map of best-fit model divided by errors in velocity. In panels (a) and (b), velocity contours are marked in white. In each panel, a red X marks the position of the black hole. We have masked out regions in order to fit only the eastern black hole. 

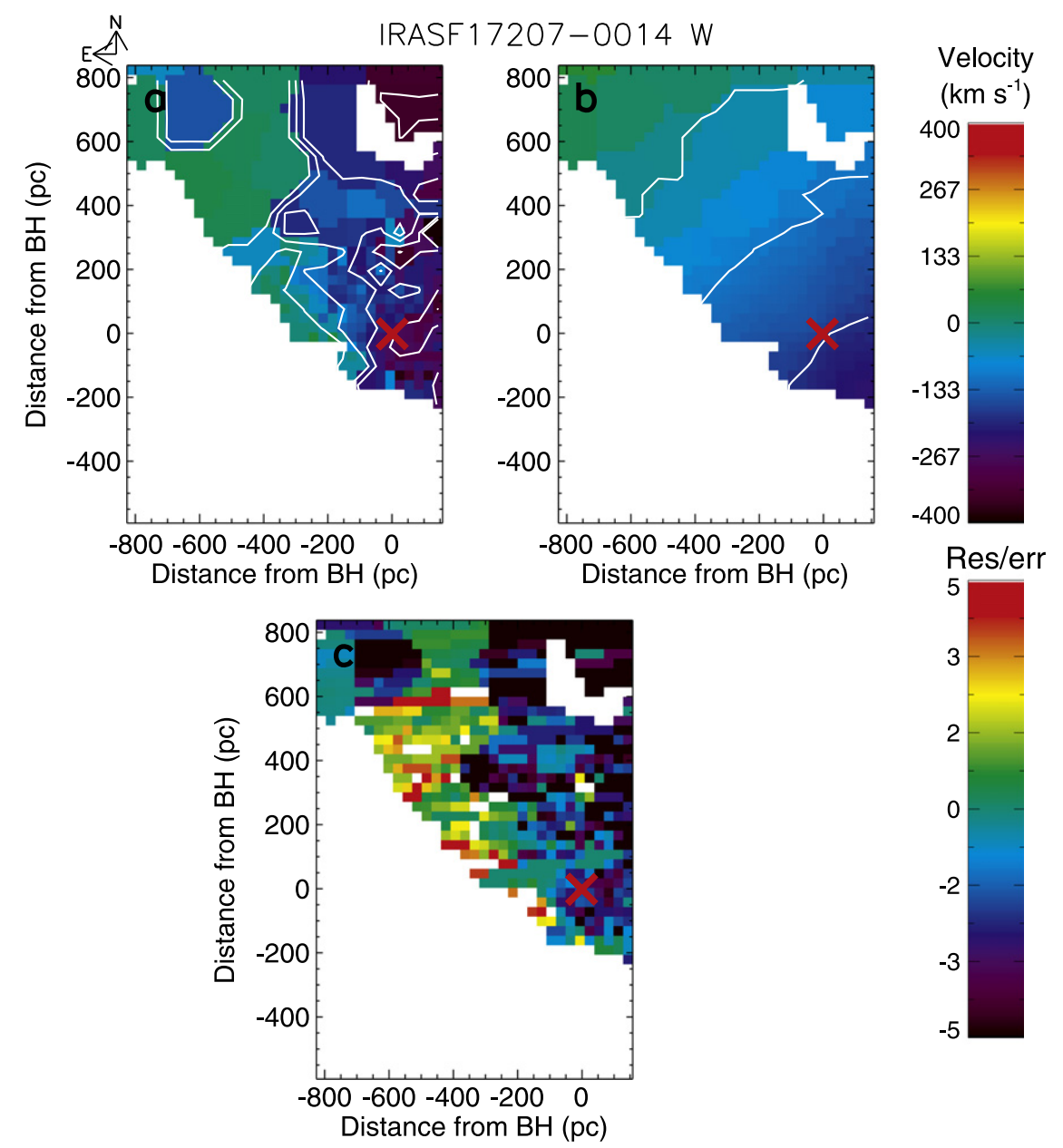

Figure A18. (a) Map of [Fe II] velocities of the inner region of IRAS F17207-0014. (b) Map of model velocities of best fit black hole model. (c) Residual map of best-fit model divided by errors in velocity. In panels (a) and (b), velocity contours are marked in white. In each panel, a red X marks the position of the black hole. We have masked out regions in order to fit only the western black hole.

Table A1

Measured Disk Parameters

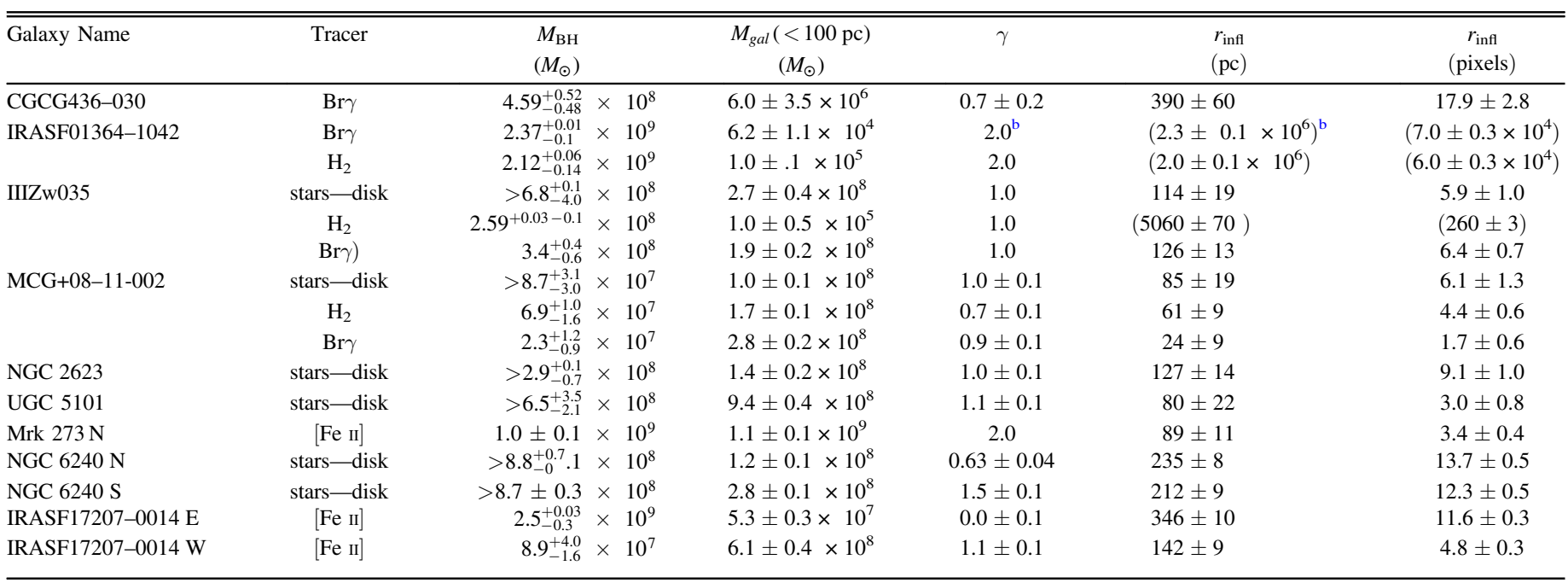

Notes.

${ }^{\mathrm{a}}$ Some fits were run with $\gamma$ fixed to match the light profile; these are thus reported with no error bars.

${ }^{\mathrm{b}}$ Radii in parentheses are artificially large because the mass of the black hole is comparable to or larger than the fitted nuclear disk. Presumably the mass profile of the galaxy changes outside of our field of view, which would reduce the radius of the sphere of influence. In the case of IIIZw035 $\mathrm{H}_{2}$, non-rotational kinematics unseen in other tracers are likely affecting the fit. 


\section{REFERENCES}

Alatalo, K., Blitz, L., Young, L. M., et al. 2011, ApJ, 735, 88 Alexander, D. M., Brandt, W. N., Smail, I., et al. 2008, AJ, 135, 1968 Armus, L., Mazzarella, J. M., Evans, A. S., et al. 2009, PASP, 121, 559 Beifiori, A., Courteau, S., Corsini, E. M., \& Zhu, Y. 2012, MNRAS, 419, 2497 Bennert, V. N., Auger, M. W., Treu, T., Woo, J.-H., \& Malkan, M. A. 2011, ApJ, 726, 59

Bondi, H. 1952, MNRAS, 112, 195

Bondi, H., \& Hoyle, F. 1944, MNRAS, 104, 273

Borys, C., Smail, I., Chapman, S. C., et al. 2005, ApJ, 635, 853

Cappellari, M. 2008, MNRAS, 390, 71

Cappellari, M., \& Copin, Y. 2003, MNRAS, 342, 345

Cappellari, M., \& Emsellem, E. 2004, PASP, 116, 138

Cen, R. 2012, ApJ, 755, 28

Cisternas, M., Jahnke, K., Bongiorno, A., et al. 2011, ApJL, 741, L11

Cisternas, M., Jahnke, K., Inskip, K. J., et al. 2011, ApJ, 726, 57

Dasyra, K. M., Tacconi, L. J., Davies, R. I., et al. 2006, ApJ, 651, 835

Davies, R. I. 2007, MNRAS, 375, 1099

Denney, K. D., Watson, L. C., Peterson, B. M., et al. 2009, ApJ, 702, 1353

Ellison, S. L., Mendel, J. T., Scudder, J. M., Patton, D. R., \& Palmer, M. J. D. 2013, MNRAS, 430, 3128

Ellison, S. L., Patton, D. R., Mendel, J. T., \& Scudder, J. M. 2011, MNRAS, 418, 2043

Ferrarese, L., \& Merritt, D. 2000, ApJL, 539, L9

Gebhardt, K., Bender, R., Bower, G., et al. 2000, ApJL, 539, L13

Gleason, J. R. 1988, Am. Stat., 42, 263

Graham, A., \& Scott, N. 2013, ApJ, 764, 151

Gültekin, K., Richstone, D. O., Gebhardt, K., et al. 2009, ApJ, 695, 1577

Haan, S., Surace, J. A., Armus, L., et al. 2011, AJ, 141, 100

Häussler, B., McIntosh, D. H., Barden, M., et al. 2007, ApJS, 172, 615

Hinshaw, G., Weiland, J. L., Hill, R. S., et al. 2009, ApJS, 180, 225

Hopkins, P. F. 2012, MNRAS, 420, L8

Hopkins, P. F., \& Elvis, M. 2010, MNRAS, 401, 7

Hopkins, P. F., Hernquist, L., Cox, T. J., et al. 2005, ApJ, 630, 705

Hopkins, P. F., Hernquist, L., Cox, T. J., et al. 2006, ApJS, 163, 1

Hopkins, P. F., Hernquist, L., Cox, T. J., Robertson, B., \& Krause, E. 2007, ApJ, 669, 45

Hopkins, P. F., Hernquist, L., Cox, T. J., Robertson, B., \& Krause, E. 2007, ApJ, 669, 67

Hopkins, P. F., Murray, N., \& Thompson, T. A. 2009, MNRAS, 398, 303

Hopkins, P. F., \& Quataert, E. 2010, MNRAS, 407, 1529

Hopkins, P. F., \& Quataert, E. 2010, MNRAS, 405, L41

Hopkins, P. F., \& Quataert, E. 2011, MNRAS, 415, 1027

Howell, J. H., Armus, L., Mazzarella, J. M., et al. 2010, ApJ, 715, 572

Ishida, C. M. 2004, PhD thesis, Univ. Hawaii

Jahnke, K., Bongiorno, A., Brusa, M., et al. 2009, ApJL, 706, L215

Kauffmann, G., \& Heckman, T. M. 2009, MNRAS, 397, 135

Kim, D.-C., Evans, A. S., Vavilkin, T., et al. 2013, ApJ, 768, 102

King, A. 2008, NewAR, 52, 253
Klöckner, H.-R., \& Baan, W. A. 2004, A\&A, 419, 887

Kocevski, D. D., Faber, S. M., Mozena, M., et al. 2012, ApJ, 744, 148

Kormendy, J., \& Gebhardt, K. 2001, in AIP Conf. Ser. 586, 20th Texas Symp.

Relativistic Astrophysics, ed. J. C. Wheeler, \& H. Martel (Melville, NY: AIP), 363

Kormendy, J., \& Ho, L. C. 2013, ARA\&A, 51, 511

Kormendy, J., \& Richstone, D. 1995, ARA\&A, 33, 581

Koss, M., Mushotzky, R., Baumgartner, W., et al. 2013, ApJL, 765, L26

Koss, M., Mushotzky, R., Veilleux, S., \& Winter, L. 2010, ApJL, 716, L125

Larkin, J., Barczys, M., Krabbe, A., et al. 2006, Proc. SPIE Conf., 6269, 62691

Magorrian, J., Tremaine, S., Richstone, D., et al. 1998, AJ, 115, 2285

Marconi, A., \& Hunt, L. K. 2003, ApJL, 589, L21

McConnell, N. J., \& Ma, C.-P. 2013, ApJ, 764, 184

Medling, A. M., Ammons, S. M., Max, C. E., et al. 2011, ApJ, 743, 32

Medling, A. M., , U. V., Guedes, J., et al. 2014, ApJ, 784, 70

Murray, N. 2009, ApJ, 691, 946

Peng, C. Y., Ho, L. C., Impey, C. D., \& Rix, H.-W. 2002, AJ, 124, 266

Peng, C. Y., Ho, L. C., Impey, C. D., \& Rix, H.-W. 2010, AJ, 139, 2097

Power, C., Nayakshin, S., \& King, A. 2011, MNRAS, 412, 269

Privon, G. C., Barnes, J. E., Evans, A. S., et al. 2013, ApJ, 771, 120

Rothberg, B., \& Joseph, R. D. 2006, AJ, 131, 185

Rupke, D. S. N., \& Veilleux, S. 2011, ApJL, 729, L27

Sanders, D. B., \& Mirabel, I. F. 1996, ARA\&A, 34, 749

Sanders, D. B., Soifer, B. T., Elias, J. H., et al. 1988, ApJ, 325, 74

Scoville, N. Z., Evans, A. S., Thompson, R., et al. 2000, AJ, 119, 991

Shier, L. M., Rieke, M. J., \& Rieke, G. H. 1996, ApJ, 470, 222

Siopis, C., Gebhardt, K., Lauer, T. R., et al. 2009, ApJ, 693, 946

Spoon, H. W. W., Farrah, D., Lebouteiller, V., et al. 2013, ApJ, 775, 127

Springel, V., di Matteo, T., \& Hernquist, L. 2005, ApJL, 620, L79

Springel, V., di Matteo, T., \& Hernquist, L. 2005, MNRAS, 361, 776

Stickley, N. R., \& Canalizo, G. 2014, ApJ, 786, 12

Sturm, E., González-Alfonso, E., Veilleux, S., et al. 2011, ApJL, 733, L16

Tecza, M., Genzel, R., Tacconi, L. J., et al. 2000, ApJ, 537, 178

Tremaine, S., Gebhardt, K., Bender, R., et al. 2002, ApJ, 574, 740

Tremonti, C. A., Heckman, T. M., Kauffmann, G., et al. 2004, ApJ, 613, 898

U., V., Sanders, D. B., Mazzarella, J. M., et al. 2012, ApJS, 203, 9

U., V., Medling, A. M., Sanders, D., et al. 2013, ApJ, 775, 115

van Dam, M. A., le Mignant, D., \& Macintosh, B. A. 2004, ApOpt, 43,5458

van Dam, M. A., Bouchez, A. H., le Mignant, D., et al. 2006, PASP, 118, 310

Veilleux, S., Kim, D.-C., \& Sanders, D. B. 2002, ApJS, 143, 315

Veilleux, S., Meléndez, M., Sturm, E., et al. 2013, ApJ, 776, 27

Wilson, C. D., Petitpas, G. R., Iono, D., et al. 2008, ApJS, 178, 189

Winge, C., Riffel, R. A., \& Storchi-Bergmann, T. 2009, ApJS, 185, 186

Wizinowich, P. L., Acton, D. S., Lai, O., et al. 2000, Proc. SPIE, 4007, 2

Wizinowich, P. L., le Mignant, D., Bouchez, A. H., et al. 2006, PASP, 118,297

Wurster, J., \& Thacker, R. J. 2013, MNRAS, 431, 2513

Wurster, J., \& Thacker, R. J. 2013, MNRAS, 431, 539

Zhang, X., Lu, Y., \& Yu, Q. 2012, ApJ, 761, 5 